Draft VERsion August 1, 2018

Preprint typeset using $\mathrm{LT}_{\mathrm{E}} \mathrm{X}$ style emulateapj v. 10/10/03

\title{
MAGNETIC FIELD EVOLUTION IN NEUTRON STAR CRUSTS DUE TO THE HALL EFFECT AND OHMIC DECAY
}

\author{
ANDREW CUMMING \\ Hubble Fellow, UCO/Lick Observatory and Department of Astronomy and Astrophysics, University of California, Santa Cruz, CA 95064
}

PHIL ARRAS

NSF AAPF Fellow, Kavli Institute for Theoretical Physics, Kohn Hall, University of California, Santa Barbara, CA 93106

ELLEN ZWEIBEL

Departments of Astronomy and Physics and Center for Magnetic Self-Organization, University of Wisconsin, 475 North Charter Street, Madison, WI 53706 Draft version August 1, 2018

\begin{abstract}
We present calculations of magnetic field evolution by the Hall effect and Ohmic decay in the crust of neutron stars (NSs). In accreting NSs, Ohmic decay is always the dominant effect due to the large resistivity. In isolated NSs with relatively pure crusts, the Hall effect dominates Ohmic decay after a time $t_{\text {switch }} \simeq 10^{4} \mathrm{yr} B_{12}^{-3}$, where $B_{12}$ is the magnetic field strength in units of $10^{12} \mathrm{G}$. We compute the evolution of an initial field distribution by Ohmic decay, and give approximate analytic formulas for both the surface and interior fields as a function of time. Due to the strong dependence of $t_{\text {switch }}$ on $B_{12}$, early Ohmic decay can alter the currents down to the base of the crust for $B \sim 10^{11} \mathrm{G}$, neutron drip for $B \sim 10^{12} \mathrm{G}$, and near the top of the crust for $B \gtrsim 10^{13} \mathrm{G}$, respectively. We then discuss magnetic field evolution by the Hall effect. Several examples are given to illustrate how an initial field configuration evolves. Hall wave eigenfunctions are computed, including the effect of the large density change across the crust. We estimate the response of the crust to the magnetic stresses induced by Hall waves, and give a detailed discussion of the boundary conditions at the solid-liquid interface. Finally, we discuss the implications for the Hall cascade proposed by Goldreich \& Reisenegger.
\end{abstract}

Subject headings: stars:magnetic fields—stars:neutron

\section{INTRODUCTION}

Our picture of neutron star magnetic field evolution comes from studies of radio pulsars, accreting neutron stars, and most recently, magnetars. The known radio pulsars fall into two general classes: young $\left(\lesssim 10^{7}\right.$ yrs $)$ pulsars with spin periods $\sim 1 \mathrm{~s}$ and $\sim 10^{12} \mathrm{G}$ magnetic fields, and the millisecond radio pulsars, which have inferred magnetic fields as low as $10^{8} \mathrm{G}$. Whereas most radio pulsars are isolated objects, the millisecond pulsars are predominantly in binaries and have undergone a period of accretion. This has led to the suggestion that millisecond pulsars form by accretion, which both spins up the neutron start to short rotation periods, and also acts to reduce the magnetic field (see Bhattacharya 1995 for a review)

This "recycling" scenario is consistent with observations of accreting neutron stars. Young accreting neutron stars in high mass X-ray binaries (HMXBs) are observed as X-ray pulsars with inferred $\sim 10^{12} \mathrm{G}$ magnetic fields, confirmed by detection of cyclotron lines in their X-ray spectra (Trümper et al. 1978). Old accreting neutron stars in low mass X-ray binaries (LMXBs) are generally not X-ray pulsars, implying no magnetic disruption of the accretion disk in those systems, and magnetic fields $\lesssim 10^{9}-10^{10} \mathrm{G}$ (see Cumming, Zweibel, \& Bildsten 2001 for a recent discussion). Recently, 5 millisecond accreting X-ray pulsars have been discovered in transient LMXBs, with spin periods between 180 and $410 \mathrm{~Hz}$, and inferred magnetic fields $10^{8}-10^{9} \mathrm{G}$, exactly in the range expected for millisecond pulsar progenitors (Chakrabarty et al. 2003)

An additional possibility is that neutron star magnetic fields decay with age. Field evolution in isolated radio pulsars has been studied using their space and velocity distributions, as well as the statistical distribution in the $P-\dot{P}$ diagram. This probes field evolution on the timescale of the ages of radio pulsars ( $\lesssim 10^{7}$ yrs $)$. At first, it was argued that exponential field decay on a few million year timescale was required to understand the $P-\dot{P}$ distribution (Ostriker \& Gunn 1969; Gunn \& Ostriker 1970; Narayan \& Ostriker 1990), but the work of Bhattacharya et al. (1992) and others in the 90's argued that in fact no field decay occurs over the lifetime of a radio pulsar. However, this issue remains somewhat open: recently, several authors have again raised the suggestion that neutron star magnetic fields evolve on a timescale something like 10 million years (Cordes \& Chernoff 1998; Tauris \& Manchester 1998; Tauris \& Konar 2001; Gonthier et al. 2002).

The discovery of magnetars has complicated this picture, but also provided a new opportunity to study magnetic field evolution on relatively short timescales. Observed as the young $\lesssim 10^{4}$ yr soft gamma repeaters (SGRs) and anomalous X-ray pulsars (AXPs), these sources are believed to be directly powered by decay of ultrastrong $\sim 10^{14}-10^{15} \mathrm{G}$ magnetic fields. Long term release of magnetic energy may act to delay the cooling of the neutron star (Thompson \& Duncan 1996; Heyl \& Kulkarni 1998; Colpi, Geppert, \& Page 2000; Arras, Cumming, and Thompson 2004), while rapid release of magnetic stresses that build in the neutron star crust (Duncan \& Thompson 1992; Thompson \& Duncan 1995, 1996) may power the frequent gamma-ray bursts observed from SGRs, and now also AXP 1E 1048.1-5937 (Gavril, Kaspi, \& Woods 2002).

These ideas and observations have motivated a large range of theoretical work on neutron star magnetic field evolution (e.g. see Bhattacharya \& Srinivasan 1995 for a review). In this paper, we discuss the evolution of currents in the neutron 
star crust, which evolve due to Ohmic decay, and by the Hall effect. The importance of currents in the crust is that they decay much more quickly than currents in the core. They therefore play an important role in models of accretion-induced field evolution: either the currents are assumed to flow only within in the crust, for example produced after the birth of the neutron star by thermomagnetic effects (Blandford, Applegate, \& Hernquist 1983), or magnetic flux may be expelled from the core into the crust due to vortex-fluxoid interactions (e.g. Srinivasan et al. 1990; Ruderman, Zhu, \& Chen 1998). At higher field strengths, Hall effects most likely are responsible for building up stresses in magnetar crusts which then are released during SGR and AXP bursts.

Previous investigations into Ohmic decay have focused mainly on the decay of the surface field, and involved calculating Ohmic decay eigenmodes for the crust (Sang \& Chanmugam 1987), self-similar solutions near the surface of the star (Urpin, Chanmugam, \& Sang 1994), or direct timeintegrations of the Ohmic diffusion equation for an initial distribution of currents (Urpin \& Muslimov 1992; Urpin \& Konenkov 1997; Page, Geppert, \& Zannias 2000). For accreting stars, the evolution due to Ohmic decay for fields supported by crustal currents has been calculated in great detail (e.g., Geppert \& Urpin 1994; Urpin, Geppert, \& Konenkov 1998; Konar \& Bhattacharya 1997). We revisit Ohmic decay in this paper, calculating both Ohmic decay modes and self-similar solutions, and show that they are related in a simple way. As well as constraining the "initial condition" for the Hall effect, this allows a general way to understand the distribution of currents in the crust at a given age, as well as the decline of the surface field.

The evolution by the Hall effect is much less wellunderstood, being non-linear and therefore much more complex. Although the Hall effect itself is non-dissipative, Goldreich \& Reisenegger (1992, hereafter GR) proposed that it leads to dissipation through a turbulent "Hall cascade", magnetic energy cascading from large to small scales where it dissipates by Ohmic decay in the Hall time associated with the largest lengthscale. Recent studies by Biskamp and collaborators (Biskamp et al. 1999) of Whistler turbulence indeed show a clear cascade of energy over more than an order of magnitude in lengthscale. Other authors have also demonstrated transfer of magnetic energy between different scales (Muslimov 1994; Naito \& Kojima 1994; Shalybkov \& Urpin 1997; Urpin \& Shalybkov 1999; Hollerbach \& Rüdiger 2002). In a different approach, Vainshtein, Chitre, \& Olinto (2000) included the effect of the density gradient in a simple model. Most recently, Rheinhardt \& Geppert (2000), Geppert \& Rheinhardt (2002), and Rheinhardt, Konenkov, \& Geppert (2003) discuss a "Hall drift instability" which can lead to nonlocal transfer of energy to small scales.

In this paper, we study the implications of realistic crust models for evolution of crustal currents. In $\$ 2$, we calculate the timescales associated with Ohmic decay and the Hall effect for realistic crust models. We discuss the relative importance of Ohmic decay and the Hall effect at different stages of a neutron star's life. In $\S 3$, we consider Ohmic decay in detail. We show that previous studies of Ohmic decay can be understood in a simple way. In $\S 4$, we discuss the evolution due to the Hall effect. We start with a simple discussion of the initial evolution of a dipole field which gives a simple physical picture. We then calculate the properties of linear Hall waves in a realistic crust model. We clarify the physical nature of the "Hall drift instability" and comment on its relevance for the overall field evolution. We summarize our main results in $\S 5$. In the Appendix, we discuss the behavior of Hall waves at the boundaries of the crust.

\section{TIMESCALES FOR THE HALL EFFECT AND OHMIC DECAY}

In this section, we estimate the timescales of evolution by the Hall effect and Ohmic decay in both isolated and accreting neutron stars, and discuss the regimes where the Hall effect is important. Both the Hall and Ohmic timescales are most sensitive to the lengthscale $L$ over which the magnetic field and crust properties vary. In this section we make a simple estimate by setting $L$ equal to the local pressure scale height $H$. This allows a comparison between the two processes, and sets the scene for the more detailed discussion in $\$ 3$ and 4

\subsection{Calculation of the Hall timescale}

The evolution of the magnetic field is given by Faraday's law,

$$
\frac{\partial B}{\partial t}=-c \nabla \times E
$$

together with Ohm's law

$$
E=-\frac{1}{c} v \times B+\frac{J}{\sigma}+\frac{J \times B}{n_{e} e c},
$$

where $\sigma$ is the electrical conductivity, and the current density is $J=(c / 4 \pi)(\nabla \times B)$ (see GR for a derivation). The first term in equation (2) describes advection of the field by the fluid, the second term describes Ohmic dissipation, and the third term is the Hall electric field ${ }^{1}$. Neglecting the advection and Ohmic terms, the magnetic field evolution is given by

$$
\frac{\partial B}{\partial t}=-\nabla \times\left(\frac{J \times B}{n_{e} e}\right),
$$

with a timescale

$$
t_{\text {Hall }}=\frac{n_{e} e L}{J}=\frac{4 \pi n_{e} e L^{2}}{c B},
$$

where $L$ is the typical lengthscale over which $B, J$, and $n_{e}$ vary. The basic physics of equation (3) is that the magnetic field is frozen to the electrons. In ideal MHD, the electron and ion velocities are the same - both follow the $E \times B$ drift. In Hall MHD, the ions follow separate dynamics. In a plasma or liquid, this only happens on scales of order the ion skindepth $\delta_{i} \equiv v_{A} / \omega_{c i}$ (or, in a high $\beta$ plasma, scales of order the ion gyroradius $\left.v_{i} / \omega_{c i}\right)$. In solids, the ions are immobilized by lattice forces and Hall effects are important on much larger scales. This is the case in the neutron star crust, which elastically adjusts as the field evolves, balancing the $J \times B$ forces with shear stresses. We discuss the issue of force balance in more detail in $\S 4$, where we consider the behavior of wavelike solutions at the boundary between the solid crust and fluid core/ocean. This is illustrated by Figure 1 which shows a variant of the usual laboratory demonstration of the Hall effect. A current-carrying conductor is placed in an external magnetic field, with the current perpendicular to the field. The conducting electrons, which move with velocity $v_{e}=-J / n_{e} e$, are deflected sideways by the magnetic field, and a Hall electric field $\mathbf{E}_{\mathbf{H}}=\mathbf{v}_{\mathbf{e}} \times \mathbf{B} / c$ develops to balance the magnetic force. The

\footnotetext{
1 We do not discuss the battery or thermoelectric terms in this paper (e.g. Blandford et al. 1983) since they are suppressed by a factor $\left(k_{B} T / E_{F, e}\right)^{2}$ for a degenerate gas, and are important in the crust only for very young neutron stars.
} 
(a)

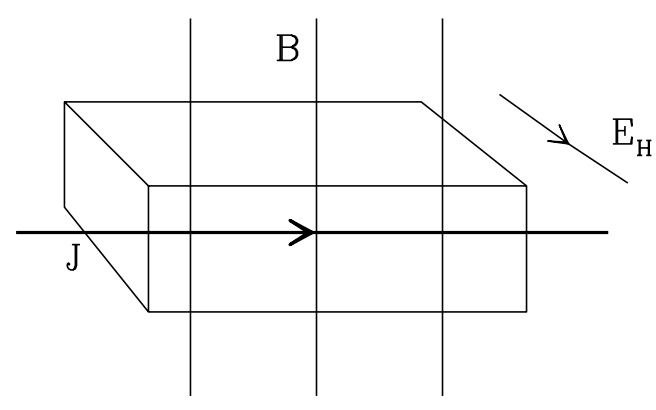

(b)

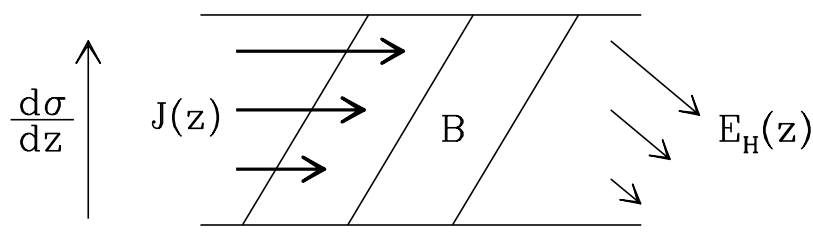

FIG. 1.- Simple example of the Hall effect: (a) the usual laboratory set up in which the Hall electric field balances the magnetic force on the conducting electrons; (b) if the conductivity varies with height, the electron velocity depends on height, shearing the magnetic field on a timescale $t_{\text {Hall }}=L / v_{e}=n_{e} e L / J$

variation on the usual experiment is to allow the conductivity to depend on height. In this case, $J$ and therefore $v_{e}$ and $E_{H}$ depend on height, giving an emf $\mathcal{E}=\int E_{H} \cdot d l$ which causes a magnetic field component to grow along the current direction. The result is that the magnetic field lines are sheared by the electron flow. In the star, this shear arises because of gradients in $B, J$, and $n_{e}$. The Hall timescale given in equation (4) is simply the shearing time of the electrons, $t_{\text {Hall }}=L / v_{e}$.

We now calculate $t_{\text {Hall }}$ for densities $\rho$ from $\approx 10^{9} \mathrm{~g} \mathrm{~cm}^{-3}$, through neutron drip at $\rho \approx 4 \times 10^{11} \mathrm{~g} \mathrm{~cm}^{-3}$, down to the crust/core interface at $\rho \approx 10^{14} \mathrm{~g} \mathrm{~cm}^{-3}$. For $\rho \lesssim$ $6 \times 10^{12} \mathrm{~g} \mathrm{~cm}^{-3}$, the relativistic, degenerate electrons dominate the pressure. The electron Fermi energy and pressure are $E_{F, e}=51 \mathrm{MeV} \rho_{12}^{1 / 3} Y_{e}^{1 / 3}$, and $P_{e}=1.2 \times$ $10^{31} \mathrm{erg} \mathrm{cm}^{-3} \rho_{12}^{4 / 3} Y_{e}^{4 / 3}$, where $\rho_{12}=\rho / 10^{12} \mathrm{~g} \mathrm{~cm}^{-3}$, and $Y_{e}$ is the number fraction of electrons. The top of the crust is set by $\Gamma=Z^{2} e^{2} / a k_{B} T=175$ (Potekhin \& Chabrier 2000), where $a=\left(3 / 4 \pi n_{i}\right)^{1 / 3}$ is the interion spacing, giving a density

$$
\rho_{\text {top }}=8 \times 10^{7} \mathrm{~g} \mathrm{~cm}^{-3} T_{8}^{3}\left(\frac{Z}{26}\right)^{-6}\left(\frac{A}{56}\right) .
$$

The top of the crust varies strongly with temperature, from $\rho \sim 10^{9} \mathrm{~g} \mathrm{~cm}^{-3}$ in accreting stars, to $\sim 10^{5} \mathrm{~g} \mathrm{~cm}^{-3}$ in isolated, cooling neutron stars.

For $\rho \gtrsim 6 \times 10^{12} \mathrm{~g} \mathrm{~cm}^{-3}$, the degenerate, non-relativistic neutrons dominate the pressure. The neutron Fermi energy and pressure are $E_{F, n}=15 \mathrm{MeV} \rho_{14}^{2 / 3} Y_{n}^{2 / 3}(f / 0.5)$, and $P_{n}=$ $6.0 \times 10^{32} \mathrm{erg} \mathrm{cm}^{-3} \rho_{14}^{5 / 3} Y_{n}^{5 / 3}(f / 0.5)$. Here, $f$ is a factor which accounts for the interactions between neutrons. We use the fit of Mackie \& Baym (1977) for $E_{F, n}$ (following Brown 2000).

The electron and neutron fractions $\left(Y_{e}\right.$ and $\left.Y_{n}\right)$ are given by the composition at each depth. An accreting neutron star can replace its crust with accreted material, in which case the composition is quite different from an isolated object. Figure 2 shows the pressure, $Y_{e}, Y_{n}$ and nuclear charge $Z$ as a function of depth in both cases. For an isolated crust, we use the cold-catalysed matter results of Haensel \& Pichon (1994) $\left(\rho<4 \times 10^{11} \mathrm{~g} \mathrm{~cm}^{-3}\right)$, and Douchin \& Haensel (2001) $(\rho>$ $\left.4 \times 10^{11} \mathrm{~g} \mathrm{~cm}^{-3}\right)$. For the accreted case, we use the results of Haensel \& Zdunik (1990, hereafter HZ), who calculated the composition changes due to electron captures and pycnonuclear reactions as fluid elements traverse the crust during accretion. HZ stop their calculation at $\rho=1.25 \times 10^{13} \mathrm{~g} \mathrm{~cm}^{-3}$; at higher densities, we fix the composition at their final value, giving $Y_{e}=0.05$ for $\rho>1.25 \times 10^{13} \mathrm{~g} \mathrm{~cm}^{-3}$ in our accreted crust. Our equation of state agrees well with Negele \& Vautherin (1973).

We show the Hall timescale as a function of density for an isolated and accreted crust in Figure 3 We choose the lengthscale $L$ to be the local pressure scale height, $H=P / \rho g$, where $g$ is the local gravity, assumed constant. For densities between $10^{12}$ and $10^{13} \mathrm{~g} \mathrm{~cm}^{-3}$, the Hall time is approximately constant with increasing density because as neutron drip occurs, the electron fraction drops dramatically, cancelling the increasing density and scale height. At lower densities, the electrons set the pressure, giving $H=77.6 \mathrm{~m} \rho_{12}^{1 / 3}\left(Y_{e} / 0.25\right)^{4 / 3}\left(2.45 / g_{14}\right)$, and

$$
t_{\text {Hall }, \text { outer }}=\frac{5.7 \times 10^{4} \mathrm{yrs}}{B_{12}} \rho_{12}^{5 / 3}\left(\frac{Y_{e}}{0.25}\right)^{11 / 3}\left(\frac{g_{14}}{2.45}\right)^{-2} .
$$

At densities greater than neutron drip, the neutrons dominate the pressure, giving

$$
\begin{aligned}
& H=245 \mathrm{~m} \rho_{14}^{2 / 3}(f / 0.5) Y_{n}^{5 / 3}\left(2.45 / g_{14}\right) \text {, and } \\
& t_{\text {Hall, inner }}=\frac{1.2 \times 10^{7} \mathrm{yrs}}{B_{12}} \rho_{14}^{7 / 3} Y_{n}^{10 / 3}\left(\frac{Y_{e}}{0.05}\right)\left(\frac{f}{0.5}\right)^{2}\left(\frac{g_{14}}{2.45}\right)^{-2} .
\end{aligned}
$$

We have inserted typical values for $Y_{e}$ at each density. In \$4 we argue that the timescale for overall evolution of the field is roughly the Hall time at the base of the crust, as given by equation (7). The choice $L=H$ introduces the $1 / g^{2}$ dependence of $t_{\text {Hall }}$ on the local gravity in the crust, and therefore some sensitivity to the equation of state of the core.

\subsection{Calculation of the Ohmic decay time}

For comparison, we now calculate the Ohmic time across a scale height $t_{\mathrm{Ohm}}=4 \pi \sigma H^{2} / c^{2}$. The conductivity is $\sigma=$ $n_{e} e^{2} / m_{\star} \nu$, or

$$
\sigma=1.52 \times 10^{25} \mathrm{~s}^{-1}\left(\frac{\nu}{10^{17} \mathrm{~s}^{-1}}\right)^{-1} \rho_{12}^{2 / 3} Y_{e}^{2 / 3},
$$

where $\nu$ is the electron scattering frequency, and $m_{\star}=$ $E_{F} / c^{2} \gg m_{e}$ is the electron effective mass, giving $t_{\mathrm{ohm}}=$ $4 \times 10^{5}$ yrs $\sigma_{25} H_{4}^{2}$.

The electrical conductivity is set by electron scattering from phonons and impurities. The phonon scattering frequency is $\nu_{e p}=\left(13 \alpha k_{B} T \Lambda_{e p} / \hbar\right)\left[1+(\Theta / 3.5 T)^{2}\right]^{-1 / 2} \exp \left(-T_{U} / T\right)$ (Baiko \& Yakovlev 1995, 1996), where $\Lambda_{e p}$ is the order unity Coulomb logarithm, and $\Theta_{D}$ is the Debye temperature,

$$
\Theta_{D}=\frac{0.45 \hbar}{k_{B}}\left(\frac{4 \pi Z^{2} e^{2} n_{i}}{A_{\text {cell }} m_{p}}\right)^{1 / 2}=3.5 \times 10^{9} \mathrm{~K} Y_{e} \rho_{12}^{1 / 2},
$$

with $A_{\text {cell }}$ the number of baryons per unit cell. Except for very young neutron stars, $T \ll \Theta_{D}$. The exponential suppression of the scattering frequency for $T<T_{U}=8.7 \times$ 


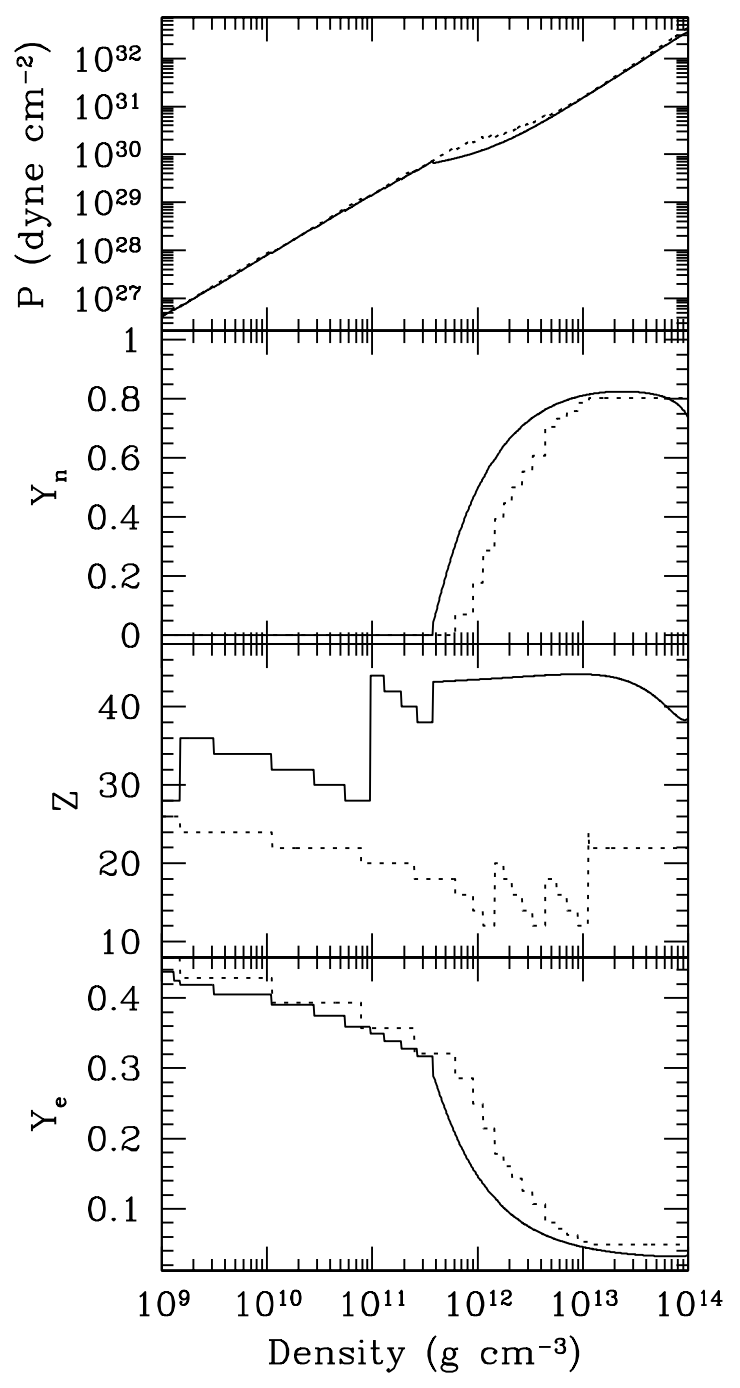

FIG. 2.- Pressure, neutron fraction $Y_{n}$, nuclear charge $Z$, and electron fraction $Y_{e}$ as a function of density for an isolated crust (solid line) and accreted crust (dotted line).

$10^{7} \mathrm{~K} \rho_{14}^{1 / 2}\left(Y_{e} / 0.05\right)(Z / 30)^{1 / 3}$ occurs because Umklapp processes freeze out below this temperature. For $T>T_{U}$, we find

$$
\sigma_{p}=\left\{\begin{array}{l}
1.2 \times 10^{24} \mathrm{~s}^{-1}\left(\rho_{12}^{7 / 6} / T_{8}^{2}\right)\left(Y_{e} / 0.25\right)^{5 / 3} \\
1.8 \times 10^{25} \mathrm{~s}^{-1}\left(\rho_{14}^{7 / 6} / T_{8}^{2}\right)\left(Y_{e} / 0.05\right)^{5 / 3}
\end{array} .\right.
$$

The impurity scattering frequency is (Itoh \& Kohyama 1993), $\nu_{e Q}=1.75 \times 10^{16} \mathrm{~s}^{-1} x Q \Lambda_{e Q} / Z$, where $x=p_{F} / m_{e} c=$ $100 \rho_{12}^{1 / 3} Y_{e}^{1 / 3}, \Lambda_{e Q}$ is the order unity Coulomb logarithm, and $Q$ is the impurity factor, giving

$$
\sigma_{Q}=\left\{\begin{array}{l}
1.6 \times 10^{25}\left(\rho_{12}^{1 / 3} / Q\right)\left(Y_{e} / 0.25\right)^{1 / 3}(Z / 30) \\
4.4 \times 10^{25}\left(\rho_{14}^{1 / 3} / Q\right)\left(Y_{e} / 0.05\right)^{1 / 3}(Z / 30)
\end{array} .\right.
$$

For clarity, we set $\Lambda_{e p}=\Lambda_{e Q}=1$ in equations (10) and (11); in our numerical calculations, we calculate these factors according to Itoh \& Kohyama (1993) and Baiko \& Yakovlev (1996), and include the exponential suppression of phonon scattering for $T<T_{U}$ as in Gnedin, Yakovlev, \& Potekhin (2001). Our conductivities agree well with those of Potekhin et al. (1999).
Impurity scattering dominates either at low temperatures $T<T_{U}$ (when phonon scattering is suppressed), or for high impurity levels $Q \gtrsim Q_{\text {crit }}$, where

$$
Q_{\text {crit }}=\left\{\begin{array}{l}
2.4\left(T_{8}^{2} / \rho_{14}^{5 / 6}\right)(Z / 30)\left(0.05 / Y_{e}\right)^{4 / 3} \\
13\left(T_{8}^{2} / \rho_{12}^{5 / 6}\right)(Z / 30)\left(0.25 / Y_{e}\right)^{4 / 3}
\end{array}\right.
$$

is given by the ratio of equations (10) and (11). For an equilibrium crust in an isolated neutron star, Flowers \& Ruderman (1977) estimated $Q \approx 10^{-3}$, in which case the conductivity is set by phonon scattering above $10^{7-8} \mathrm{~K}$, depending on position in the crust, and by impurity scattering at lower temperatures. However, recent calculations by Jones (2001) suggest a much larger value $Q \sim 10$, in which case impurity scattering may set the conductivity even for $T>10^{8} \mathrm{~K}$.

In accreting neutron stars, the crust temperature is high $\left(\gtrsim 10^{8} \mathrm{~K}\right)$ for rapid accretors $\left(\gtrsim 10^{-11} M_{\odot} \mathrm{yr}^{-1}\right)$, in which case phonons dominate for $Q \lesssim \mathcal{O}(1)$. However, $Q$ is set by the composition of the nuclear burning which occurs at low densities, and is likely to be $\gtrsim \mathcal{O}(1)$ (for example, Schatz et al. 1999 found $Q \approx 100$ ), in which case impurities dominate.

When impurities dominate, the Ohmic time is

$$
\begin{array}{r}
t_{\mathrm{Ohm}}=5.7 \operatorname{Myrs} \frac{\rho_{14}^{5 / 3}}{Q}\left(\frac{Z}{30}\right)\left(\frac{Y_{e}}{0.05}\right)^{1 / 3}\left(\frac{Y_{n}}{0.8}\right)^{10 / 3} \\
\left(\frac{f}{0.5}\right)^{2}\left(\frac{g_{14}}{2.45}\right)^{-2},
\end{array}
$$

and when phonons dominate, it is

$$
\begin{array}{r}
t_{\mathrm{Ohm}}=2.2 \operatorname{Myrs} \frac{\rho_{14}^{15 / 6}}{T_{8}^{2}}\left(\frac{Y_{e}}{0.05}\right)^{5 / 3}\left(\frac{Y_{n}}{0.8}\right)^{10 / 3} \\
\left(\frac{f}{0.5}\right)^{2}\left(\frac{g_{14}}{2.45}\right)^{-2} .
\end{array}
$$

The ohmic time $t_{\mathrm{Ohm}}=4 \pi \sigma L^{2} / c^{2}$ is shown in Figure 3 for equilibrium crusts with $Q=10^{-3}$, and $T=10^{6} \mathrm{~K}$ and $10^{8} \mathrm{~K}$, and for an accreted crust with $T=5 \times 10^{8} \mathrm{~K}$ and $Q=1$.

\subsection{When does the Hall Effect dominate Ohmic decay?}

The ratio of Ohmic time $t_{\mathrm{ohm}}$ to the Hall time $t_{H}$ is equivalent to the product of electron cyclotron frequency and electron collision time, $\Omega \tau=e B \tau / m_{\star} c$. Large $\Omega \tau$ means that $t_{\mathrm{Hall}} \ll t_{\mathrm{Ohm}}$, and the Hall effect dominates. If the conductivity is set by impurities $\left(Q>Q_{\text {crit }}\right.$ or $\left.T<T_{U}\right)$

$$
\Omega \tau=\left\{\begin{array}{l}
7\left(B_{12} / Q \rho_{12}^{2 / 3}\right)(Z / 30)\left(Y_{e} / 0.25\right)^{-2 / 3} \\
1\left(B_{12} / Q \rho_{14}^{2 / 3}\right)(Z / 30)\left(Y_{e} / 0.05\right)^{-2 / 3}
\end{array},\right.
$$

and if the conductivity is set by phonons $\left(Q<Q_{\text {crit }}\right.$ and $T>$ $\left.T_{U}\right)$

$$
\Omega \tau=\left\{\begin{array}{l}
0.6\left(B_{12} \rho_{12}^{1 / 6} / T_{8}^{2}\right)\left(Y_{e} / 0.25\right)^{2 / 3} \exp \left(T_{U} / T\right) \\
0.4\left(B_{12} \rho_{14}^{1 / 6} / T_{8}^{2}\right)\left(Y_{e} / 0.05\right)^{2 / 3} \exp \left(T_{U} / T\right)
\end{array},\right.
$$

These different regimes are summarized in Figure 4 We show the $\Omega \tau=1$ contour in the $B-T$ plane for conditions near the base of the crust $\left(\rho=10^{14} \mathrm{~g} \mathrm{~cm}^{-3}\right)$. Note, however, that $\Omega \tau$ is reasonably insensitive to $\rho$, so that all depths change from Ohmic decay-dominated to Hall-dominated at roughly the same time.

Figure 4 shows very clearly the difference in evolution between isolated and accreting neutron stars. An isolated neutron star cools to $T<T_{U}$ in $\approx 1 \mathrm{Myr}$, after which impurities 

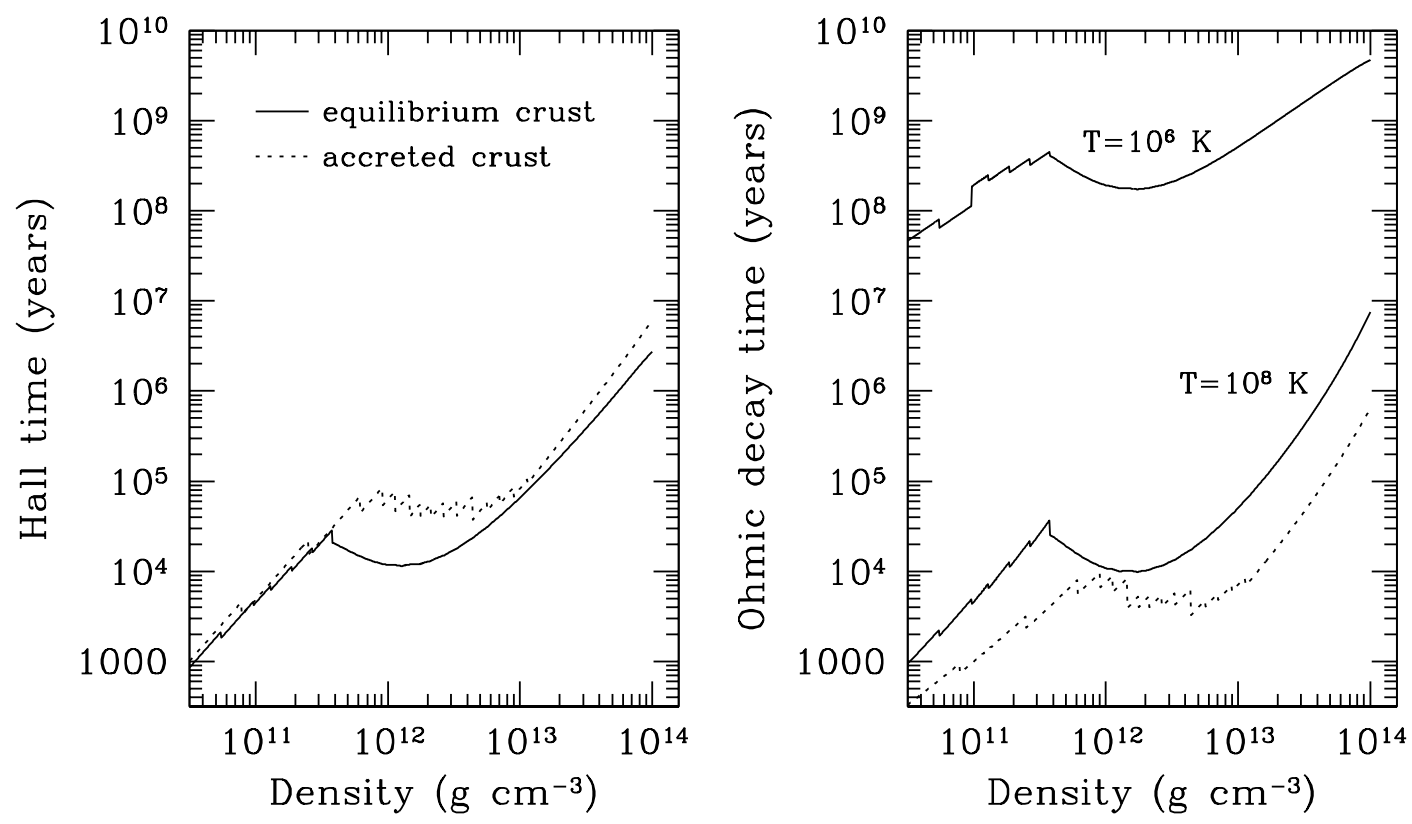

FIG. 3.- Hall timescale $t_{\text {Hall }}$ (left panel) and ohmic time $t_{\mathrm{ohm}}$ (right panel) as a function of density for two examples of an equilibrium crust (solid lines) and accreted crust (dashed line). The equilibrium crusts have $Q=10^{-3}$ and $T=10^{6} \mathrm{~K}$ and $10^{8} \mathrm{~K}$; the accreted crust has $T=5 \times 10^{8} \mathrm{~K}$ and $Q=1$. We assume the crust is isothermal, with a constant magnetic field $B=10^{12} \mathrm{G}\left(t_{\text {Hall }} \propto 1 / B\right)$, and take the relevant lengthscale to be the scale height, with local gravity $g=2.45 \times 10^{14} \mathrm{~cm} \mathrm{~s}^{-2}$.

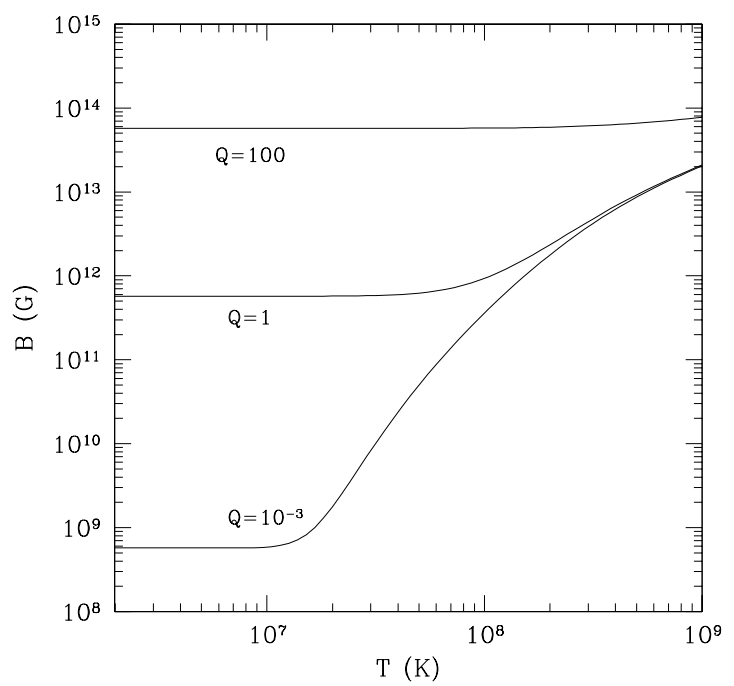

FIG. 4.- Ohmic decay vs. Hall effect. The solid lines show the $B$ field above which $\Omega \tau \geq 1$ as a function of temperature at a density of $\rho=10^{14} \mathrm{~g} \mathrm{~cm}^{-3}$, and for an isolated crust.

set the conductivity giving $\Omega \tau \simeq B_{12} / Q$ at the base. The extent to which Hall effects dominate then depends sensitively on the value of $Q$ for an isolated crust. The recent calculation by Jones (2001) of the formation enthalpies of point defects in the crust gives $Q \gtrsim 1$ at densities greater than neutron drip (see also de Blasio 2000 for densities lower than neutron drip). In this case Ohmic decay dominates the evolution even at late times. The original estimates of Flowers \& Ruderman (1977) gave $Q \ll 1$, in which case the Hall effect dominates for $T<T_{U}$. An accreting neutron star has $T>T_{U}$ because of heating by accretion (e.g. Brown 2000), and in addition may have a large impurity parameter $Q>1$ because of the complex mixture of nuclei produced by burning of the accreted hydrogen and helium (Schatz et al. 1999). This means that whether impurities or phonons dominate, the conductivity is poor enough that $\Omega \tau \ll 1$, implying that the Hall effect is not important for an accreting star (even after accretion terminates, if the crust is impure).

For an isolated neutron star with a pure crust $(Q \ll 1)$, Figure 4 allows us to estimate when the Hall effect will dominate Ohmic decay. For $Q=10^{-3}$ at the base of the crust, the critical temperature below which the Hall effect is dominant is $T \approx 6 \times 10^{7}, 2 \times 10^{8}$, and $6 \times 10^{8} \mathrm{~K}$ for magnetic fields $B=10^{11}, 10^{12}$ and $10^{13} \mathrm{G}$, respectively. At these high temperatures, $T \geq T_{U}$ and the conductivity is set by electron-phonon scattering. To estimate the core temperature as a function of time, we adopt the cooling law for modified URCA neutrino emission with specific heat given by normal (unpaired) neutrons, $t \simeq 10^{6}$ yr $T_{8}^{-6}$ (e.g. Shapiro and Teukolsky 1983). Using equation (16), the age at which the Hall effect becomes dominant is

$$
t_{\text {switch }} \simeq 2 \times 10^{4} \mathrm{yr} B_{12}^{-3} .
$$

Ohmic decay is more important in lower field stars since the Hall effect takes over at a later time. For typical pulsar magnetic fields, the Hall effect starts to dominate while the star is still quite young. Ohmic decay calculations ignoring the Hall effect are only valid for $t<t_{\text {switch }}$.

\section{FIELD EVOLUTION WHEN $\Omega \tau \ll 1$}

In this section, we compute the Ohmic decay of currents in the crust at early times when $\Omega \tau \ll 1$, before the Hall effect dominates the field evolution. We first calculate Ohmic decay 
modes, and then discuss the Green's function solution, which shows self-similar behavior at late times. We show that these solutions are simply related, giving a general way to understand the distribution of currents in the crust at a given age, as well as the decline of the surface field. This constrains the "initial condition" for the later evolution by the Hall effect.

\subsection{Ohmic decay modes}

We now calculate the Ohmic decay modes for currents confined to the crust. We write the poloidal magnetic field in terms of the potential $f(r, t), B=\nabla \times \nabla \times\left[f(r, t) Y_{l}^{m}(\theta, \phi)\right]$ (Chandrasekhar 1961). The field components are

$$
B=\frac{l(l+1)}{r^{2}} f Y_{l}^{m} e_{r}+\frac{1}{r} \frac{d f}{d r}\left(\frac{\partial Y_{l}^{m}}{\partial \theta} e_{\theta}+\frac{\partial Y_{l}^{m}}{\partial \phi} e_{\phi}\right) .
$$

Faraday's law (eq. [1]) with $E=J / \sigma$ gives

$$
\frac{\partial f}{\partial t}=\eta(r, t)\left(\frac{\partial^{2} f}{\partial r^{2}}-\frac{l(l+1)}{r^{2}} f\right)
$$

where $\eta=c^{2} / 4 \pi \sigma$ is the magnetic diffusivity.

When Ohmic decay determines the evolution of the field, the conductivity is mainly set by phonons, in which case the time-dependence of $\eta$ arises only through the temperature, $\eta \propto T^{2}$. Following Urpin et al. (1994), we absorb this time-dependence into a new time variable $\hat{t}$, given by $d \hat{t} \propto T^{2} d t$. For modified URCA cooling $T \propto t^{-1 / 6}$, we find $t=(\hat{t} / 1.5 \mathrm{Myr})^{3 / 2}$ Myr when $\eta$ is evaluated at the reference temperature $T_{8}=1$. We then look for Ohmic decay modes, $f \propto \exp (-\hat{t} / \tau)$, giving

$$
\frac{d^{2} f}{d r^{2}}-\frac{l(l+1)}{r^{2}} f=-\frac{f}{\eta \tau} .
$$

Note that exponential decay in $\hat{t}$ represents a slower decay in real time $t$, because the conductivity of the cooling crust grows with time, reducing the Ohmic dissipation rate.

Equation (20) is an eigenvalue problem for $\tau$ given boundary conditions for $f$. At the top of the crust, we match onto a vacuum field, giving $f \propto r^{-l}$, and normalize the field such that $B_{r}=1$ there. At the base of the crust, we consider two different boundary conditions, either $B_{r}=0$ at the base, implying the field is confined entirely to the crust, or we assume the field connects to an interior vacuum field, an example of a field which threads the neutron star core ${ }^{2}$.

A WKB analysis clarifies the basic properties of the modes. Writing $f \propto \exp \left(i \int^{r} d r k_{r}\right) \exp (-\hat{t} / \tau)$, we find the dispersion relation

$$
k_{r}^{2}=\frac{1}{\eta \tau}-\frac{l(l+1)}{r^{2}} \simeq(\eta \tau)^{-1}
$$

where $k_{r}$ is the radial wavenumber and $\tau$ is the decay time. We ignore the horizontal wavenumber term, which is negligible unless $l \geq R / H_{b} \simeq 20$. The WKB solution, including amplitude and phase, is

$$
f \propto \eta^{1 / 4} \exp \left( \pm i \int^{r} \frac{d r}{(\eta \tau)^{1 / 2}}\right) .
$$

\footnotetext{
${ }^{2}$ In fact, if the field penetrates the core, then there is no strict boundary condition quantizing the phase in units of roughly $n \pi$ in the crust. The vertical wavelength in the core will be much smaller than in the crust, and then the value and slope at the crust core interface will reflect quantizing the phase in

\begin{tabular}{|c|c|c|}
\hline$n$ & $t_{\mathrm{Ohm}} / \tau_{n}$ & $t_{\mathrm{Ohm}} / n^{2} \tau_{n}$ \\
\hline \multicolumn{3}{|c|}{$T_{8}=1, Q=10^{-3}, t_{\mathrm{Ohm}}=7.5 \times 10^{6} \mathrm{yrs}$} \\
\hline 1 & 3.60 & 3.60 \\
\hline 2 & 18.2 & 4.56 \\
\hline 3 & 44.0 & 4.89 \\
\hline 4 & 82.0 & 5.12 \\
\hline 5 & 133.0 & 5.33 \\
\hline \multicolumn{3}{|c|}{$T_{8}=3, Q=10^{-3}, t_{\mathrm{Ohm}}=7.0 \times 10^{5} \mathrm{yrs}$} \\
\hline 1 & 2.32 & 2.32 \\
\hline 2 & 12.1 & 3.03 \\
\hline 3 & 29.8 & 3.31 \\
\hline 4 & 55.8 & 3.49 \\
\hline 5 & 90.4 & 3.61 \\
\hline \multicolumn{3}{|c|}{$T_{8}=0.01, Q=10^{-3}, t_{\mathrm{Ohm}}=4.7 \times 10^{9} \mathrm{yrs}$} \\
\hline 1 & 0.315 & 0.315 \\
\hline 2 & 2.17 & 0.542 \\
\hline 3 & 5.67 & 0.630 \\
\hline 4 & 10.8 & 0.675 \\
\hline 5 & 17.6 & 0.703 \\
\hline \multicolumn{3}{|c|}{$\begin{array}{c}T_{8}=1, Q=10^{-3}, t_{\mathrm{Ohm}}=7.5 \times 10^{6} \mathrm{yrs} \\
\text { inner dipole boundary condition }\end{array}$} \\
\hline 1 & 0.0729 & 0.0729 \\
\hline 2 & 8.76 & 2.19 \\
\hline 3 & 28.8 & 3.20 \\
\hline 4 & 60.5 & 3.78 \\
\hline 5 & 105 & 4.20 \\
\hline
\end{tabular}
the core. For eigenmodes with many nodes in the crust, this will not change the waves significantly, but for long decay times $\gg 10^{6} \mathrm{yr}$, this boundary condition is crucial.
}

Table 1. Ohmic Decay Modes (Dipole)

For $f$, the amplitude and wavelength grow $\propto \eta^{1 / 2}$ toward the top of the crust. The magnetic field and current components have the same oscillatory phase, but their envelopes scale as $B_{r} \propto \eta^{1 / 4}, B_{\theta, \phi} \propto \eta^{-1 / 4}$, and $J_{\theta, \phi} \propto \eta^{-3 / 4}$. A WKB turning point is reached at the depth $z_{t p}(\tau)$ at which $k_{z} H_{\sigma} \sim 1$, or equivalently, where the mode decay time equals the local diffusion time. Above this depth, the left and right sides of equation 20 can no longer balance, leading to evanescent behavior.

We show two examples of Ohmic decay eigenmodes with $l=1$ in Figures $5\left(B_{r}=0\right.$ at the base) and 6 (vacuum field at the base). The left panel in each case shows the lowest order eigenmode. When $B_{r}=0$ at the base, $B_{r}$ varies rapidly with depth, giving $B_{\theta} \approx(R / H) B_{r} \gg B_{r}$ at the base; for a vacuum boundary condition at the base, $B_{r}$ varies only slightly across the crust, giving $B_{r} \sim B_{\theta}$ at the base. For short wavelength modes (right panels), the eigenfunctions become insensitive to the lower boundary condition; the effect of the boundary condition is to introduce an overall phase shift. The WKB amplitude scalings and turning point can be seen clearly for these modes.

Table 1 gives Ohmic decay times for numbers of nodes $n=1-5$, different temperatures, impurity fractions and inner boundary conditions. The results are scaled in terms of the Ohmic time at the base, which is listed in the table. For $T=10^{8} \mathrm{~K}$, the decay times for the lowest order modes are $\tau_{1}=2.1 \times 10^{6} \mathrm{yr}$ and $\tau_{1}=1.0 \times 10^{8} \mathrm{yr}$ for the $B_{r}=0$ and vacuum inner boundary conditions. As pointed out by Pethick \& Sahrling (1995), the difference between these two cases is that the lowest order mode has a decay time $\approx R / H$ times larger when the field penetrates into the core, because the lengthscale on which $B_{r}$ changes through the crust is the radius instead of the crust thickness.

\subsection{Green's function solution}



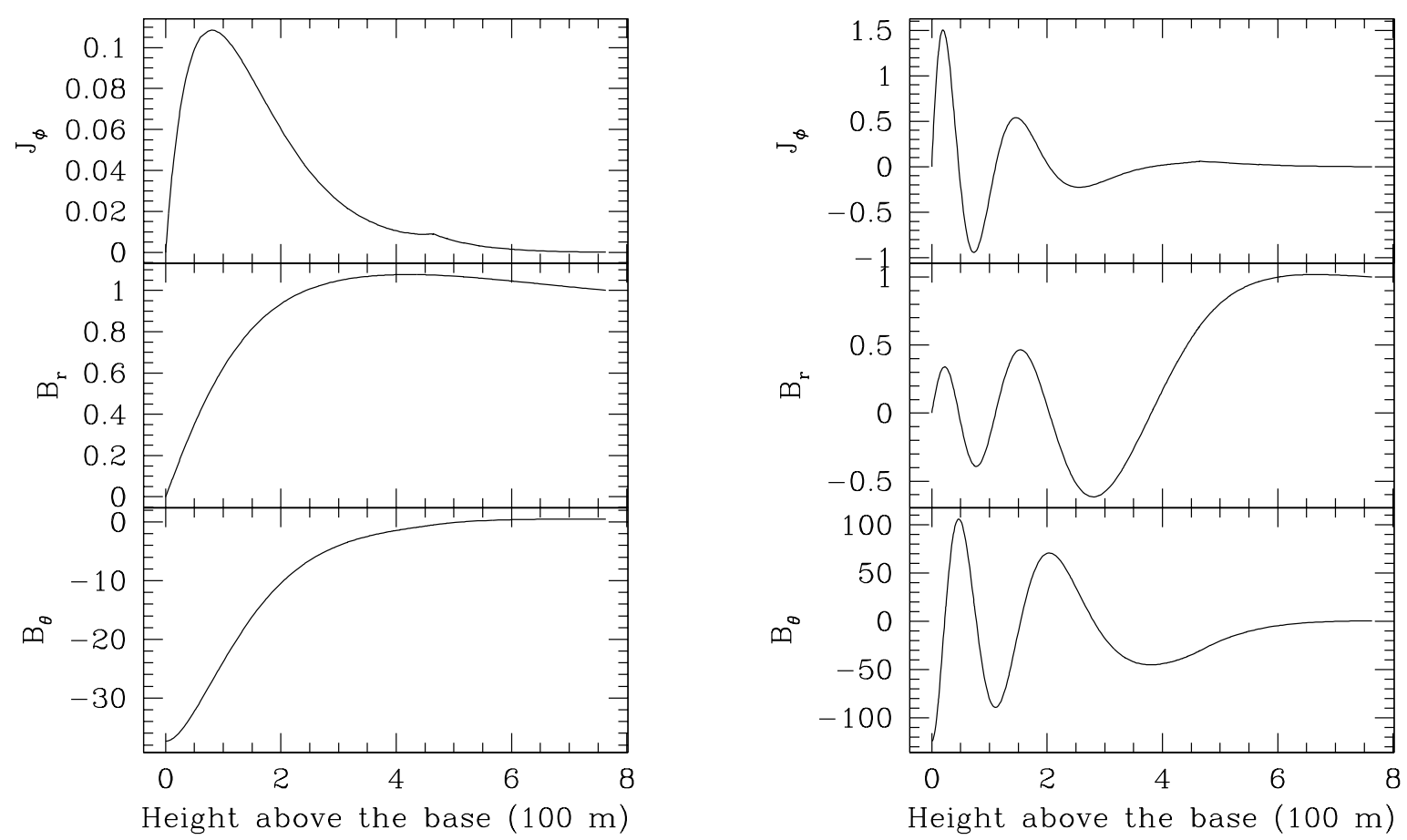

FIG. 5.- Ohmic decay mode eigenfunctions for an equilibrium crust with $T=10^{8} \mathrm{~K}$ and $Q=10^{-3}$. We show the lowest order mode ( $\left.n=1\right)$ in the left panel and the $n=5$ mode in the right panel. The inner boundary condition is $B_{r}=0$.
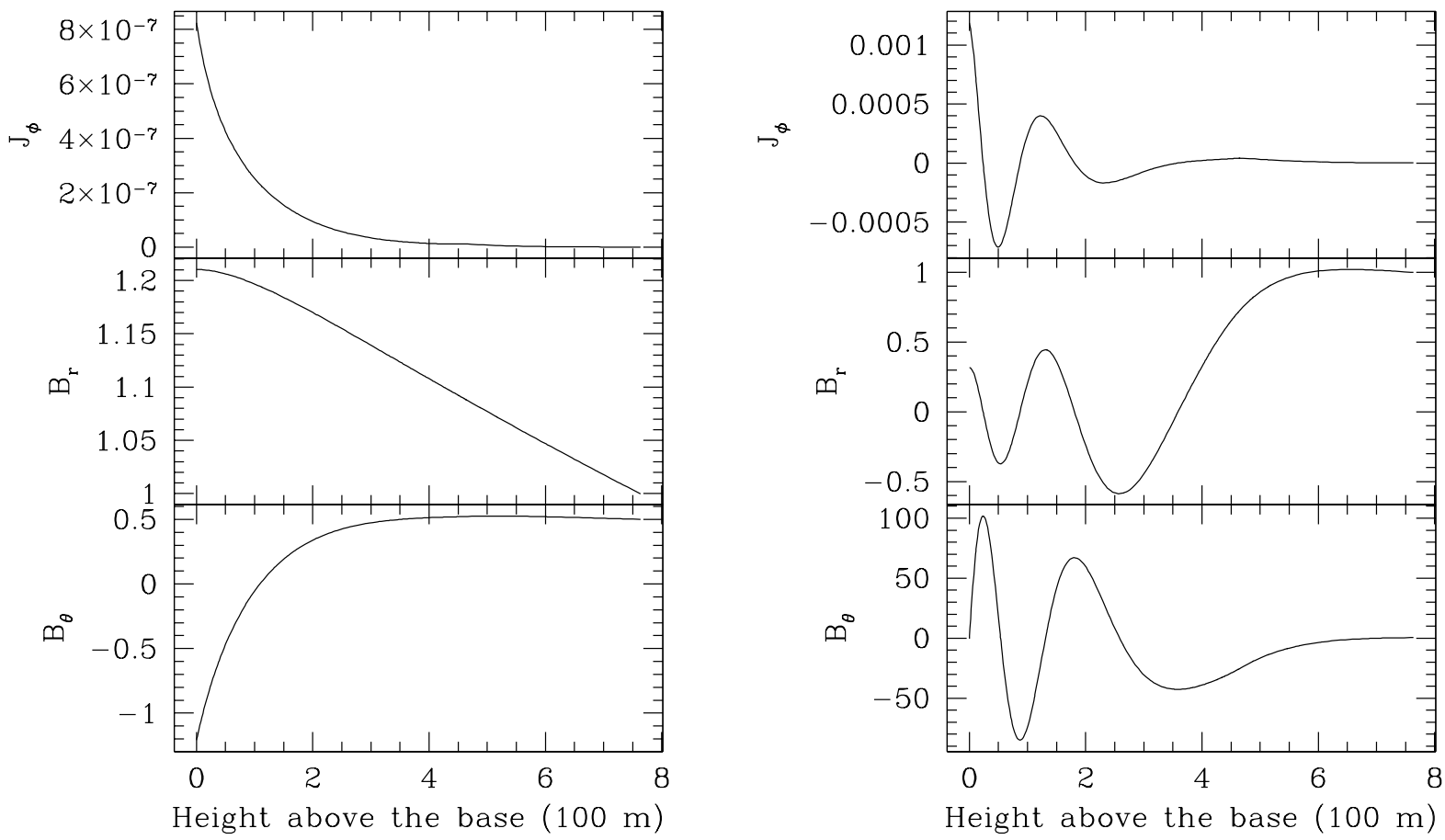

FIG. 6.- Same as Figure 5 but with an inner dipole boundary condition.

An alternative method of solving the diffusion equation is to find the Green's function. We now calculate the eigenmodes and Green's function for a plane parallel crust with power- law diffusivity $\eta \propto z^{-\alpha}$ (where $z$ is the depth). ${ }^{3}$. We start by

\footnotetext{
${ }^{3}$ Although we choose a power law diffusivity mainly because it allows an analytic solution, this is also a good approximation since the conductivity is a power law in both position and time over a large range of scales.
} 


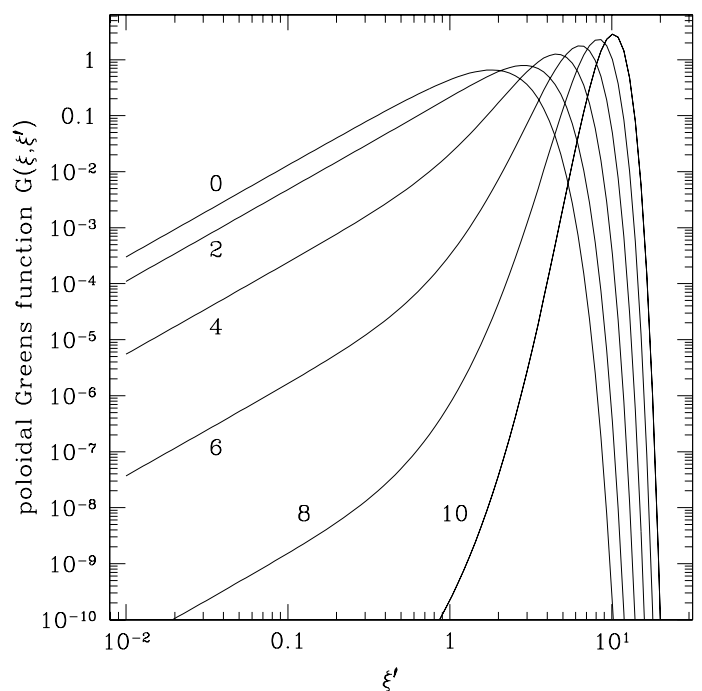

FIG. 7.- Green's function for Ohmic decay of poloidal magnetic field. The conductivity profile is $\alpha=7 / 2$. Each line is labelled with the value of $\xi$.

defining the similarity variable

$$
\xi(z, \hat{t})=\int_{0}^{z} \frac{d z}{\sqrt{\eta \hat{t}}} .
$$

Useful quantities are $\hat{t}_{\text {diff }}(z)=\xi^{2} \hat{t} \propto z^{\alpha+2}$, the characteristic diffusion time to depth $z$, and $z_{\mathrm{diff}}(\hat{t}) \propto \hat{t}^{1 /(\alpha+2)}$, the depth for which the diffusion time is the current age, $\hat{t}=\hat{t}_{\text {diff }}$, given by $\xi\left(z_{\text {diff }}, \hat{t}\right)=1$.

Setting $\eta \propto z^{-\alpha}$, we look for solutions $\propto f(\xi) \exp (-\hat{t} / \tau)$, where $\xi=\xi(z, \tau)$, giving

$$
f(\xi)=\xi^{\nu} J_{-\nu}(\xi)
$$

where $\nu=(\alpha+2)^{-1}$, and $J_{-\nu}$ is a Bessel function of the first kind. The solution with order $-\nu$ becomes constant near the surface, therefore matching onto a vacuum field at low densities. The decay time $\tau$ for each mode is determined by enforcing the boundary condition at the base. These eigenmodes have similar properties to those determined in $\$ 3.1$. The turning point for each mode occurs at $\xi \sim 1$, or depth $z_{\text {diff }}(\tau)$. Since the amplitude of $J_{\nu}(\xi)$ is $\propto \xi^{-1 / 2}$ for $\xi \gg 1$, we find $f \propto z^{-\alpha / 4} \propto \eta^{1 / 4}$ at large depths, as expected from the WKB scalings (eq. [22]).

The Green's function is obtained by writing an initial delta function as a sum over the eigenmodes given by equation (24), and using the Fourier-Bessel inversion formula (Jackson 1975, pg. 100) and a standard integral over Bessel functions (Watson 1966, pg. 395). We find the Green's function solution (see also Eichler \& Cheng 1989)

$$
f(\xi, \hat{t})=\int d \ln \xi^{\prime} G\left(\xi, \xi^{\prime}\right) f_{0}\left(z^{\prime}\right)
$$

where

$$
G(\xi, \xi \prime)=\frac{1}{2} \xi^{\nu}\left(\xi^{\prime}\right)^{2-\nu} I_{-\nu}\left(\frac{\xi \xi \prime}{2}\right) \exp \left(-\frac{\xi^{2}+\xi^{2}}{4}\right),
$$

$\xi=\xi(z, \hat{t}), \xi \prime=\xi(z \prime, \hat{t}), I_{\nu}$ is a modified Bessel function, and $f_{0}(z)$ is the initial field profile. For $\xi \ll 1, G$ becomes independent of $\xi$,

$$
G \propto\left(\xi^{\prime}\right)^{2(1-\nu)} \exp \left(-\frac{\xi^{\prime 2}}{4}\right)
$$

Figure 7] shows the Green's function for $\alpha=7 / 2$ and $\nu=2 / 11$, appropriate for electron-phonon conductivity above neutron drip.

The evolution in time can be understood by taking various limits of equation (25). Consider the case where the currents extend down to a finite depth $z_{\text {init }}$ initially, $f_{0}\left(z>z_{\text {init }}\right)=0$. For early times (or sufficiently large depths), the field is unchanged: $G \approx \delta\left(\xi^{\prime}-\xi\right)$ for $\xi \gg 1$, giving

$$
f(z, \hat{t}) \simeq f_{0}(z) \quad \xi \gg 1, z>z_{\mathrm{diff}}(\hat{t})
$$

For late times $(\xi \ll 1)$, equation $(27)$ applies, and the solution is independent of depth, with a time-dependence determined by the integral over $\xi^{\prime}$. When $z_{\text {diff }}(\hat{t})<z_{\text {init }}$, the peak of the Green's function at $\xi^{\prime} \approx 1$ gives

$$
f(z, \hat{t}) \simeq f_{0}\left(z_{\text {diff }}(\hat{t})\right) \quad \xi \ll 1, z_{\text {diff }}(\hat{t})<z_{\text {init }},
$$

so that the solution is determined by the initial value of the field at the current diffusion depth $z_{\text {diff }}(\hat{t})$. If $f_{0}$ varies slowly with depth, then $f$ will vary slowly with time during this phase, particularly since $z_{\text {diff }}$ is a weak power of $\hat{t}$. When $z_{\text {diff }}(\hat{t})>z_{\text {init }}$, however, the integrand drops to zero at a value of $\xi^{\prime}<1$, so that equation (27) gives $G \propto\left(\xi^{\prime}\right)^{2(1-\nu)}$. Writing the solution as an integral over $z^{\prime}$, the time-dependence factors out as

$$
f(z, \hat{t}) \propto \hat{t}^{\nu-1} \propto \hat{t}^{-(\alpha+1) /(\alpha+2)} \quad \xi \ll 1, z_{\text {diff }}(\hat{t})>z_{\text {init }} .
$$

Therefore at late times, the field decays as a power law in time, independent of the initial field profile (Sang \& Chanmugam 1987). Since $\hat{t} \propto t^{2 / 3}$, we find that the surface field decays as

$$
\frac{B(t)}{B_{0}}=\left(\frac{t_{\text {diff }}\left(z_{\text {init }}\right)}{t}\right)^{\delta},
$$

with $\delta=0.55(\alpha=7 / 2)$ for $z_{\text {init }}$ above neutron drip, and $\delta=0.49(\alpha=7 / 4)$ for $z_{\text {init }}$ below neutron drip. For completeness, we also note that when impurity scattering dominates the conductivity, $\hat{t} \propto t$ and $\alpha \simeq 4 / 3$, giving $\delta=0.7$.

The connection between the eigenmode and Green's function solutions is now easily seen. The Green's function solution implies that $f$ is constant for $z<z_{\text {diff }}(\hat{t})$, and unchanged for $z>z_{\text {diff }}(\hat{t})$. In terms of Ohmic modes, at a given time $\hat{t}$, all modes with decay times $\tau \ll \hat{t}$, which propagate closer to the surface than $z_{\text {diff }}(\hat{t})$, have exponentially decayed away. Therefore for $z<z_{\text {diff }}(\hat{t})$ the solution is dominated by the mode with $\tau=\hat{t}$ which has turning point at $z_{\text {diff }}(\tau)$. The power law decay of the surface field at late times arises because the decay time of the mode which dominates the surface field increases with time (Sang \& Chanmugam 1987). For $z>z_{\text {diff }}(\hat{t})$, the field profile is a superposition of all WKB modes with decay times $\tau \geq \hat{t}$.

\subsection{Toroidal fields}

Toroidal fields behave in a qualitatively similar way to poloidal fields, but with some important quantitative differences. Defining the toroidal field as $B=\nabla \times\left(g(r) Y_{l}^{m} e_{r}\right)$ (Chandrasekhar 1961), we find the diffusion equation is

$$
\frac{\partial g}{\partial t}=\frac{\partial}{\partial r}\left(\eta \frac{\partial g}{\partial r}\right)-\eta \frac{l(l+1)}{r^{2}} g,
$$

with WKB solution

$$
g \propto \eta^{-1 / 4} \exp \left( \pm i \int^{z} \frac{d z}{(\eta \tau)^{1 / 2}}\right)
$$




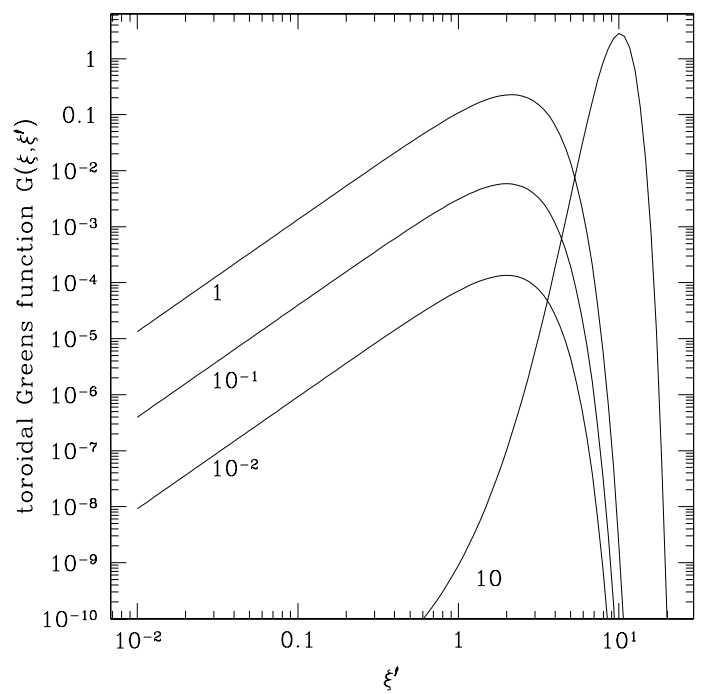

FIG. 8. - Green's function for Ohmic decay of toroidal magnetic field. The conductivity profile is $\alpha=7 / 2$.Each line is labelled with the value of $\xi$.

giving $B_{\theta, \phi} \propto \eta^{-1 / 4}, J_{\theta, \phi} \propto \eta^{-3 / 4}$, and $J_{r} \propto \eta^{-1 / 4}$. These scalings are similar to the poloidal case. For diffusivity $\eta \propto z^{-\alpha}$, the power-law solution has the same form as in equation (24), but with $\nu=(\alpha+1) /(\alpha+2)$, and the Bessel function must have order $+\nu$ rather than $-\nu$ to ensure zero field at the boundary. The toroidal Green's function is plotted in Figure 8 The major difference from the poloidal case is for late times, for which the field decays $\propto \hat{t}^{-(2 \alpha+3) /(\alpha+2)} \propto t^{-1.2}$ (for $\alpha=7 / 2$ ), much faster than the poloidal case (compare eq. [30]). In addition, the field decreases rapidly towards the surface, $g \propto \xi^{2 \nu} \propto z^{\alpha+1}$, implying the interior toroidal fields are much larger than those near the surface.

\subsection{Summary of Ohmic decay}

The results of this section give a very simple way to understand the distribution of currents and the decay of the surface dipole field given the age of the star. For a general conductivity profile $\eta(z)$, the rescaled time corresponding to the upper turning point is

$$
\hat{t}_{\text {diff }}(z)=\left(\int_{0}^{z} \frac{d z}{\eta^{1 / 2}(z)}\right)^{2},
$$

where $z=0$ is at the top of the crust. We plot the physical time $t_{\text {diff }} \propto \hat{t}_{\text {diff }}^{3 / 2}$ for a detailed crust model with electron-phonon scattering in Figure 9 This plot can be read as follows: since we have included the fact that the temperature is decreasing in time, set the ordinate $t_{\text {diff }}$ equal to the age $t$, and then read off the density at which $z=z_{\text {diff }}$. At lower densities, the currents have decayed according to equation (31), and we expect $f$ approximately constant, dominated by the Ohmic decay mode with $\tau \approx t$; at higher densities, the initial field has not yet evolved significantly, and is dominated by modes with $\tau>t$. We emphasise again that we assume phonons dominate the conductivity in Figure 9 This is a good assumption even if the crust is impure $Q \gtrsim 1$, except at the very base of the crust (see eq. [12]). After $\approx 1 \mathrm{Myr}$, the crust conductivity becomes impurity scattering dominated, and the field decay slows dramatically if $Q \ll 1$.

Our results compare well with the time-dependent calculations of Urpin and collaborators (Urpin \& Muslimov 1992;

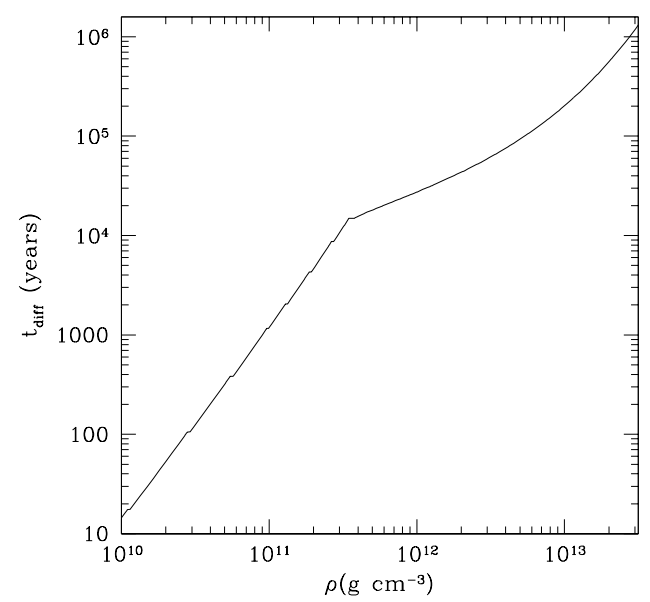

FIG. 9.- Ohmic time corresponding to the upper turning point as a function of distance from the top of the crust. We assume phonon conductivity and modified URCA cooling ( $T \propto t^{-1 / 6}$ ) (for $t \gtrsim 1 \mathrm{Myr}$, impurity scattering dominates the conductivity, in which case the Ohmic evolution slows considerably for $Q \ll 1$ ). (i) For a given age $t$, this plot shows the density at which currents are just beginning to evolve due to Ohmic diffusion. At lower densities, the field is roughly constant with depth, dominated by the Ohmic decay mode with $\tau \approx t$; at higher densities, the field has not yet evolved significantly, and is dominated by Ohmic decay modes with $\tau>t$. (ii) For currents initially distributed up to a density $\rho_{\text {init }}$, this plot gives the time at which the surface field evolution changes from roughly constant with time to a power law $\propto t^{-0.55}$.

Urpin \& Konenkov 1997; Page et al. 2000). Their initial profile $f_{0}(z)$ varies slowly with depth in the crust, giving very little decay of the field for $t<t_{\text {diff }}\left(z_{\text {init }}\right)$ (eq. [29]). For $t>t_{\text {diff }}\left(z_{\text {init }}\right)$, the decay of the observable dipole field agrees well with equation (31). We do not reproduce the much longer ( $\sim 10^{8}$ year) decay timescales found by Chamugam \& Sang (1987); the reason for this discrepancy is not clear.

At $t=t_{\text {switch }}$ (eq. [17) when the Hall effect begins to dominate Ohmic decay, we find that the currents must reside at a density $\gtrsim 10^{10} \mathrm{~g} \mathrm{~cm}^{-3}$ (towards the top of the crust) for $B=10^{13} \mathrm{G}, \gtrsim 10^{12} \mathrm{~g} \mathrm{~cm}^{-3}$ (near neutron drip) for $B=10^{12} \mathrm{G}$, and $\gtrsim 10^{14} \mathrm{~g} \mathrm{~cm}^{-3}$ (near the base of the crust) for $B=10^{11} \mathrm{G}$. Therefore the distribution of currents when the Hall effect begins to dominate the evolution depends sensitively on the field strength.

\section{FIELD EVOLUTION WHEN $\Omega \tau \gg 1$}

In this section, we first give a qualitative discussion of how the Hall effect acts on the magnetic field. We discuss how an initial large lengthscale poloidal dipole field generates higher wavenumbers, and discuss an interesting time-independent solution of the Hall equation. Next, we present short lengthscale Hall wave solutions for realistic crust models, including a first calculation of the elastic response of the crust to the wave. We discuss the Hall drift instability found by Rheinhardt \& Geppert (2002), and finally discuss the implications of our results for GR's non-linear Hall cascade.

\subsection{Qualitative discussion of Hall evolution}

In terms of the electron velocity $v_{e}=-J / n_{e} e$, the induction equation (3) is

$$
\frac{\partial B}{\partial t}=\nabla \times\left(v_{e} \times B\right) .
$$


Although this shows a formal relation to the usual MHD advection equation, it differs because the electron velocity depends directly on $B$. Nonetheless, it is useful to think of the field moving with the electron fluid in the same way as the field is tied to fluid elements in MHD. It is instructive to rewrite equation (35) as

$$
\frac{D B}{D t}=B \cdot \nabla v_{e}-B \frac{v_{e, r}}{H_{e}}
$$

where $D / D t=\partial / \partial t+v_{e} \cdot \nabla$ is an advective derivative following the electrons, $v_{e, r}$ is the radial component of the electron velocity, and $H_{e}$ is the electron density scale height. The first term on the right hand side of equation (36) represents shearing of the field by the electron flow; the second term arises because $\nabla \cdot v_{e}$ is non-zero for a vertical current because of the background electron density gradient: $\nabla \cdot J=0$ gives $\nabla \cdot v_{e}=-v_{e} \cdot \nabla n_{e} / n_{e}$. For example, as currents flow to deeper regions, the electron density increases, the electron velocity decreases, and the field is amplified by compression.

As a first example, consider an axisymmetric poloidal field $B_{P}=B_{r}(r, \theta) e_{r}+B_{\theta}(r, \theta) e_{\theta}$, supported by toroidal currents which we write in terms of the electron angular velocity $\Omega_{e}$ as $v_{e}=\Omega_{e}(r, \theta) r \sin \theta e_{\phi}$. In this case, equation (35) gives $\partial B_{P} / \partial t=0$, and

$$
\frac{\partial B_{\phi}}{\partial t}=r \sin \theta\left(B_{P} \cdot \nabla\right) \Omega_{e},
$$

showing that any differential rotation of the electron flow along the poloidal field lines shears the field, generating a toroidal component and a net twist. This is a direct ana$\log$ of the winding up of field lines by differential rotation (e.g. Mestel 1999). Figure 10 a) shows an initial dipole field and associated toroidal current. In this case, shear in the electron velocity at mid-latitudes generates a toroidal field shown in Figure 10 (b). The net effect is to twist the original field in one direction in the northern hemisphere and the opposite direction in the southern hemisphere. The subsequent evolution is determined by the poloidal currents associated with $B_{\phi}$. They act back on the original poloidal field, "pinching" it at the equator, and generating a quadrupole component of the poloidal field, as shown in Figure 10 (c). In this way, the process continues, and the smaller and smaller scale components of the field develop.

As a second example, we consider an axisymmetric toroidal field $B_{T}=B_{\phi}(r, \theta) e_{\phi}$, supported by poloidal currents $v_{e}=$ $v_{r}(r, \theta) e_{r}+v_{\theta}(r, \theta) e_{\theta}$. In this case, equation (35) gives

$$
\frac{\partial B_{\phi}}{\partial t}=-v_{e} \cdot \nabla B_{\phi}-B_{\phi} \frac{v_{e, r}}{H_{e}}+B_{\phi}\left(\frac{v_{e, r}}{r}+\frac{v_{e, \theta} \cot \theta}{r}\right),
$$

and $\partial B_{P} / \partial t=0$, so that an initially toroidal field remains toroidal. The terms on the right hand side of equation (38) describe advection by poloidal currents, divergence of the velocity flow due to the electron density gradient, and spherical geometry effects. Vainshtein et al. (2000) solved equation (38) for a plane-parallel geometry, in which case it reduces to Burger's equation, since only the term involving $H_{e}$ survives. For a toroidal field with opposite signs in the northern and southern hemispheres, as in Figure 10 b), they showed that the toroidal field strength grows at the equator, where the electrons flow inwards to higher density, and is reduced in strength towards the pole, where the electrons flow outwards to lower density (or vice versa depending on the overall field direction). They argued that if the field propagates towards (a)

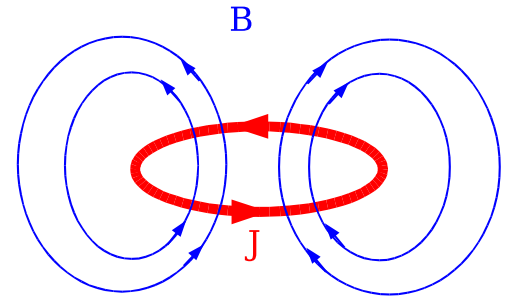

(b)

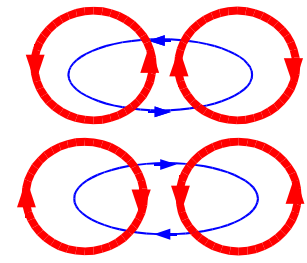

(c)

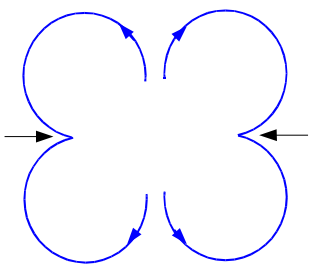

FIG. 10.- The first steps in the evolution of an initial dipole field by the Hall effect. (a) The toroidal current supporting the dipole field shears the field at mid-latitudes. (b) The resulting toroidal field and associated poloidal currents. The net effect is to "twist" the original dipole field. (c) The poloidal currents "pinch" the original poloidal dipole field, generating a poloidal quadrupole.

the equator, a thin current sheet will form, rapidly dissipating the magnetic energy. It is not clear whether this effect persists in spherical geometry and including a poloidal component of the field.

These examples show that the field geometry can give a large correction to the Hall timescale estimate of $\$ 2.1$ For example, in Figure 10 the initial evolution of the field is by shearing $B_{r}$ at mid-latitudes. If the magnetic field is confined to the crust, we expect $B_{r} \approx B_{\theta}(H / R)$ to vary across a lengthscale $H$, and $J_{\phi} \approx(c / 4 \pi)\left(B_{\theta} / H\right)$. This gives $t_{\text {Hall }}=$ $4 \pi n_{e} e H^{2} / c B_{\theta}$, so that the horizontal field is the appropriate field to use in the Hall time estimates of $\$ 2.1$ If the magnetic field penetrates into the core, then we expect $B_{r} \approx B_{\theta}$, and $J_{\phi} \approx(c / 4 \pi)\left(B_{\theta} / R\right)$ giving a Hall timescale a factor $R / H$ longer. This is directly analogous to the sensitivity of the lowest order Ohmic decay mode to the boundary condition at the base that we found in $\$ 3$

\subsection{Time-Independent Solutions}

Not all magnetic field configurations evolve under the Hall effect. The existence of time-independent solutions may be important for two reasons. First, it implies that dissipation by the Hall effect is not necessarily able to cause the field to decrease to arbitrarily small values (assuming that the role of the Hall effect is to drive dissipative processes on small scales). Second, time-independent solutions may act as a background on which Hall waves propagate. The most obvious timeindependent solution is a force free field with $J \times B=0$, or $J=\kappa B$, where $\kappa$ is constant along a given field line (e.g. Mestel 1999). The field profile in this case may or may not reflect lengthscales associated with the crust, depending on the initial relaxation of the field when the neutron star is born.

An intriguing time-independent solution is suggested by equation (37). For an axisymmetric dipole field, the toroidal current is $J_{\phi} \propto \sin \theta$ (e.g. Fig. 10a), implying that $\Omega_{e} \propto v_{e} / \sin \theta$ is constant, or the electrons rotate rigidly on spherical shells. Equation (37) then becomes $\partial B_{\phi} / \partial t=$ $r \sin \theta B_{r}\left(\partial \Omega_{e} / \partial r\right)$. A simple time-independent solution is therefore $\Omega_{e}=$ constant, or rigid rotation of the electrons! If the electrons rigidly rotate, no shearing of the poloidal field occurs, and therefore no evolution due to the Hall effect. This 
solution implies $J_{\phi} \propto n_{e}$, and therefore large currents running at the base compared to the top of the crust. If this current is sufficiently large compared to currents in the core, then deep in the crust the field is $B_{\theta} \simeq-\left(4 \pi J_{\phi, b} H_{e} / c\right)\left(n_{e} / n_{e, b}\right)$, $B_{r} \simeq 2 B_{\theta}\left(H_{e} / r\right)$, where $J_{\phi, b}$ and $n_{e, b}$ are the current and electron density at the base. This solution is not force free, but implies an increasing magnetic force with depth; the magnetic stresses are poloidal however, and hence may be supported in the fluid above and below the crust.

It is interesting to speculate whether further timeindependent solutions exist. Equation (37) suggests, analogous to Ferraro's law of isorotation (Ferraro 1937; Mestel 1999), further steady-states in which the electrons rigidly rotate along each poloidal field line. However, the difference between the Hall effect and the MHD case is that the electron velocity depends directly on the magnetic field. The poloidal dipole field is special because the currents are such that no differential rotation of the electrons occurs on spherical shells. This is not true in general, and therefore the dipole case may be the only steady-state solution of this kind. A toroidal field in plane-parallel geometry $B=B(x, z) \hat{e}_{y}$ (where we assume the field is "axisymmetric", $\partial / \partial y=0)$, and with constant $n_{e}$, does not evolve due to the Hall term, since $\nabla \times(J \times B)=0$. However, Vainshtein et al. (2000) noted that a gradient in $n_{e}$ gives rise to compression of the field (second term on the right hand side of eq. [38]), and concluded that the field is not steadystate in general. This is likely also true once the extra terms arising from spherical effects (eq. [38]) are included, unless the angular distribution of the extra terms can be arranged to match (and cancel) the compression term.

The time independent solution is stable to small perturbations. In order to see this, let $B$ and $J$ be the magnetic field and current density of the time independent state, and $\delta B$ and $\delta J$ be the corresponding perturbations of this state. Linearizing eq. (3) with respect to the perturbations gives

$$
\frac{\partial \delta B}{\partial t}=-\nabla \times\left(\frac{J \times \delta B+\delta J \times B}{e n_{e}}\right) .
$$

Taking the scalar product of eq. (39) with $\delta B / 4 \pi$ and integrating over the volume of the crust gives an equation for the magnetic energy in the perturbation

$$
\frac{\partial}{\partial t} \int \frac{\delta B^{2}}{8 \pi} d V=\int \frac{J}{e n_{e} c} \cdot(\delta J \times \delta B) d V .
$$

In deriving eq. 40, we have integrated the right hand side once by parts, used Ampere's Law, and assumed the perturbations vanish on the boundary. Next, we rewrite $\delta J \times \delta B / c$ as the divergence of the Maxwell tensor

$$
\frac{\delta J \times \delta B}{c}=\nabla \cdot T
$$

where

$$
T \equiv \frac{\delta B \delta B}{4 \pi}-I \frac{\delta B^{2}}{8 \pi} .
$$

We need only the $T_{i \phi}$ components of eq. (42) because $J$ has only a $\phi$ component; $J / e n_{e}=-\hat{\phi} \Omega_{e} r \sin \theta$, with $\Omega_{e}$ a constant. Writing out the integrand on the RHS of eq. (40), using eqs. (41) and (42), and applying Gauss's Theorem, we find that the integral vanishes identically. This shows that in the absence of any dissipation, small perturbations to the steady state neither grow nor decay. Although the realizability of this steady state is open to question, the demonstration that it is stable certainly bolsters its case for relevance.
Lastly, we mention that this background solution will decay with time at a rate given roughly by the Ohmic time at the base of the crust (section 2.2).

\subsection{Hall waves}

To make further progress, we consider the evolution of perturbations to a background magnetic field. GR showed that such perturbations propagate as circularly-polarized Hall waves. For constant electron density and a uniform magnetic field, the induction equation describing plane-wave perturbations $\delta B \propto \exp (i k \cdot x)$ is

$$
\frac{\partial \delta B}{\partial t}=\frac{c k \cdot B}{4 \pi n_{e} e}(k \times \delta B),
$$

which may be derived by perturbing equation (3) (see also Thompson \& Duncan 1996). The dispersion relation is

$$
\omega=\frac{c k|k \cdot B|}{4 \pi n_{e} e}=\nu k\left|k \cdot \hat{e}_{B}\right|,
$$

where we define $\nu \equiv c B / 4 \pi n_{e} e=\omega_{c i} \delta_{i}^{2} \propto B / n_{e}$. GR discussed the suggestion by Jones (1988) that Hall waves could transport energy to lower densities in the crust, where Ohmic dissipation is more efficient. They pointed out that as the wave moves to lower $n_{e}$, the vertical wavelength must increase (for fixed $\omega$; see eq. [44]), implying a reflection or turning point for the wave when $k_{z} H \sim 1$. In this section, we calculate the properties of Hall waves in a realistic crust model. We determine the location of the turning point as a function of wavelength. In addition, we calculate the strain induced by the Hall wave in the crust, and discuss unstable modes in the presence of a background electron velocity gradient.

\section{Force balance in the solid}

The Hall wave in the crust relies on the fact that the solid can adjust to accommodate the $J \times B$ forces that arise as the wave propagates (see also Franco et.al. (2000) for the case of magnetic stresses induced by spin down). To see this, consider perturbations $\propto \exp (-i \omega t+i k \cdot x)$ of a uniform background field with constant $n_{e}$ as before, but now include the shear stress (with constant shear modulus $\mu$ ) and the ion inertia. Since we assume constant $n_{e}$, the orientation of the field is not important. The induction equation (43) has an extra term $\nabla \times(\delta v \times B)$, giving

$$
-i \omega \delta B=\frac{c k \cdot B}{4 \pi n_{e} e}(k \times \delta B)+k \times(\omega \boldsymbol{\xi} \times B),
$$

where we write the velocity in terms of the fluid displacement as $\delta v=i \omega \boldsymbol{\xi}$. The momentum equation is

$$
-\rho \omega^{2} \boldsymbol{\xi}=\frac{\delta J \times B}{c}-\mu k^{2} \boldsymbol{\xi}
$$

with the terms representing the ion inertia, magnetic force, and shear stress. Solving for $\omega$, we find the dispersion relation

$$
\omega^{2}=\frac{\omega_{\text {Hall }}^{2}\left(\omega^{2}-\omega_{s}^{2}\right)}{\left(\omega^{2}-\omega_{s}^{2}-\omega_{A}^{2}\right)},
$$

where $\omega_{s}^{2}=k^{2} \mu / \rho$ and $\omega_{A}^{2}=(k \cdot B)^{2} / 4 \pi \rho$ define the shear and Alfven wave frequencies, and $\omega_{\text {Hall }}=c k(k \cdot B) / 4 \pi n_{e} e$ is the Hall wave frequency.

In the solid crust, which has a finite shear modulus $\omega_{s}^{2}>0$, there is a low frequency $\left(\omega^{2} \ll \omega_{A}^{2}, \omega_{s}^{2}\right)$ solution to equation (47),

$$
\omega=\frac{\omega_{\text {Hall }}}{1+B^{2} / 4 \pi \mu} \text {. }
$$


For $B^{2} / 4 \pi \ll \mu$, we recover the usual Hall wave: the induction and momentum equations are uncoupled. For $B^{2} / 4 \pi \gg \mu$, the emf induced by the fluid displacements is large enough to counteract the Hall emf, reducing the Hall wave frequency. For typical pulsar fields, this is an important effect only at the very top of the crust; for magnetar-strength fields, it is an important effect throughout much of the crust. The shear modulus is $\mu \approx 0.1 n_{i}(Z e)^{2} / a$ (Strohmayer et al. 1991), where $a=\left(3 / 4 \pi n_{i}\right)^{1 / 3}$ is the interion spacing ${ }^{4}$, giving $B^{2} / 4 \pi \mu=$ $5 \times 10^{-3} B_{12}^{2}\left(\rho_{9} Y_{e}\right)^{-4 / 3}(Z / 26)^{-2 / 3}$. In this paper, we assume $B^{2} \ll 4 \pi \mu$, so that the induction and momentum equations are uncoupled in the crust.

In the fluid above or below the crust, the shear modulus is zero, so that the $J \times B$ force must be balanced by the ion inertia, giving a natural wave frequency $\omega \approx \omega_{A} \gg \omega_{\text {Hall }}$. One might expect that the Hall waves would be almost completely reflected at the crust/fluid boundaries because of this extremely large mismatch in wave frequency. However, deriving the behavior of the strain $\theta=d \xi / d z$ near the boundaries requires a careful treatment of the boundary conditions. In this paper, we calculate the response of the crust for the simplest case of a vertically propagating wave on a vertical background field. We discuss the behavior of the wave at the boundary in the Appendix. The key point is that the Hall term enforces continuity of the magnetic perturbation across the boundary. Therefore, the magnetic stress cannot abruptly change to accommodate the loss of shear stress on moving into the fluid. This implies that $\mu \theta \approx B \delta B / 4 \pi \approx 0$ at the fluid/solid interface, which gives complete reflection.

\section{Vertically-propagating waves including the density gradient}

We now include the electron density gradient, and study vertically-propagating Hall waves in a uniform vertical background field. In terms of helicity eigenstates $\delta B_{ \pm}(z)=$ $\delta B_{x}(z) \pm i \delta B_{y}(z)$, the perturbed induction equation is

$$
\frac{d}{d z}\left(\nu \frac{d \delta B_{ \pm}}{d z}\right) \pm \omega \delta B_{ \pm}=0
$$

Since $\boldsymbol{\nabla} \cdot \boldsymbol{\delta} B=0, \delta B_{z}=$ constant for these modes.

We integrate equation (49) through the crust, using a shooting method to find the eigenvalues $\omega$ for which $\delta B=0$ at each fluid/solid interface. Figures 11, 13 show the eigenmodes with $n=1,10$, and 100 nodes, normalized so that the maximum perturbation is $\delta B=10^{12} \mathrm{G}$.

The properties of the eigenmodes can be simply understood with a WKB analysis. A good fit to the electron density profile in the crust is $n_{e}(z) \simeq n_{e, b}\left(z / 4 H_{b}\right)^{4}$, with $n_{e, b}=3 \times 10^{36} \mathrm{~cm}^{-3}$ and $H_{b} \simeq 250 \mathrm{~m}$ the electron density and scale height at the base of the crust. Using this to quantize the number of nodes $n$ over the crust, $\int k_{z} d z=n \pi$ gives the wave period

$$
P_{\text {Hall }} \simeq \frac{t_{\text {Hall, }}}{n^{2}} \simeq \frac{10^{7} \mathrm{yrs}}{B_{12} n^{2}}
$$

The WKB solution gives $\delta B \propto k^{1 / 2} \propto n_{e}^{1 / 4} \propto z$, in good agreement with the numerical solutions. The wave reaches a turning point when $\int k_{z} d z \sim 1$, or at a depth $z_{\text {turn }} \approx 3 H_{b} / n^{1 / 3}$. In terms of density, the turning point is

$$
\rho_{\text {turn }} Y_{e} \approx \frac{2 \times 10^{12} \mathrm{~g} \mathrm{~cm}^{-3}}{n^{4 / 3}},
$$

\footnotetext{
${ }^{4}$ A convenient way to write this when relativistic degenerate electrons provide the pressure is $\mu / P_{e} \approx 10^{-2}(Z / 26)^{2 / 3}$.
}

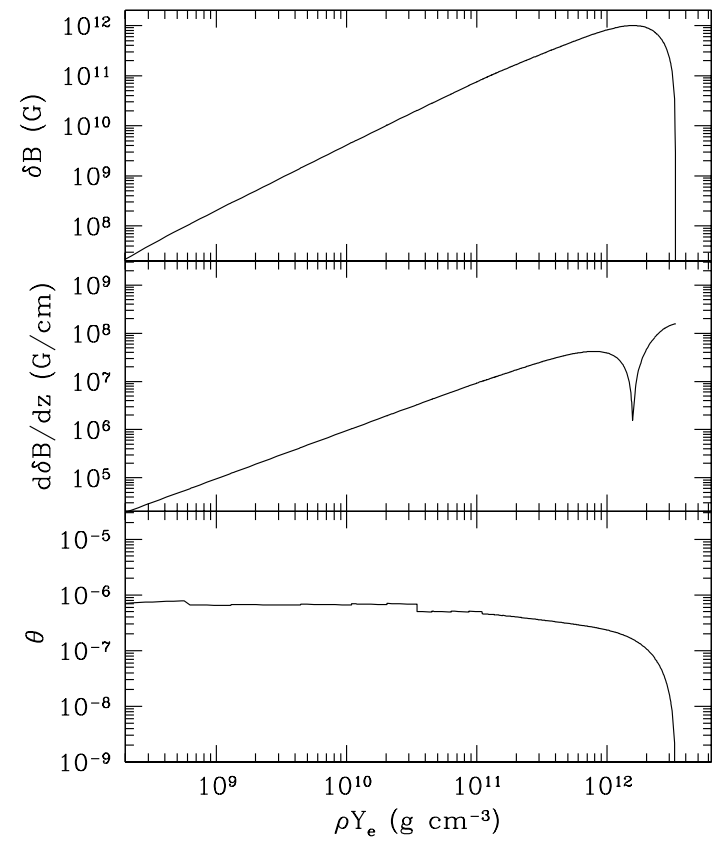

FIG. 11.- Hall wave with no nodes in a constant vertical background field of $B=10^{12} \mathrm{G}$. This wave has a period $P_{1}=4.4 \times 10^{6} \mathrm{yr} B_{12}^{-1}$. We normalize the wave so that it has a maximum amplitude $\delta B / B=1$. At the top of the crust (not shown), $\theta$ drops rapidly to zero (see text).

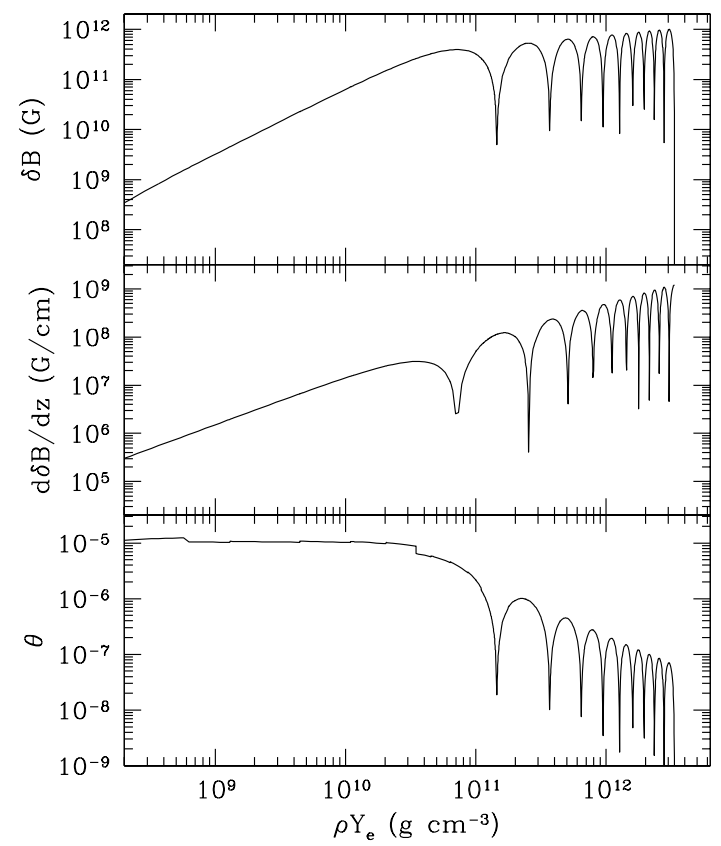

FIG. 12.- As Figure 11 but for 10 nodes. This wave has a period $P_{10}=$ $5.7 \times 10^{4}$ yr $B_{12}^{-1}$.

which requires $n \approx 300$ for $\rho_{\text {turn }} \approx 10^{9} \mathrm{~g} \mathrm{~cm}^{-3}$, or $n \approx 3 \times 10^{5}$ for $\rho_{\text {turn }} \approx 10^{5} \mathrm{~g} \mathrm{~cm}^{-3}$. Above the turning point, $\delta B$ decreases to match the boundary condition $\delta B=0$. Since $\nu \propto z^{-4}$, equation (49) gives a steep dropoff $\delta B \propto z^{5}$, in agreement with our numerical results. 


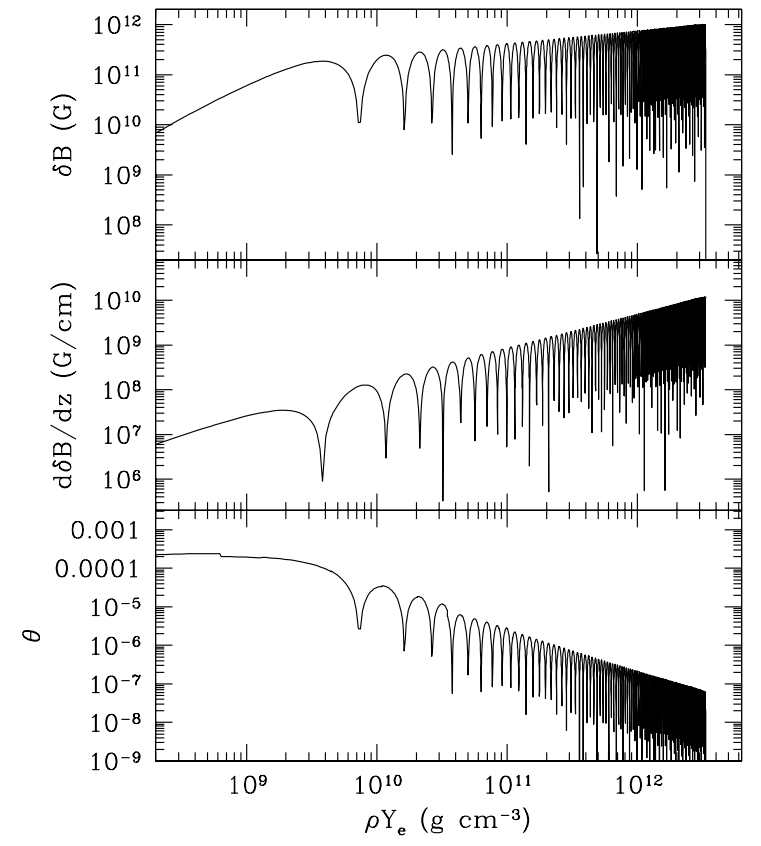

FIG. 13.- As Figure 11 but for 100 nodes. This wave has a period $P_{100}=580$ yr $B_{12}^{-1}$.

The Ohmic dissipation rate for the mode is

$$
\begin{aligned}
\gamma & =\int d V \frac{|\delta J|^{2}}{\sigma} / \int d V \frac{|\delta B|^{2}}{4 \pi} \\
& \simeq \int_{z_{t p}}^{z_{b}} \frac{d z}{v_{g, z}} \frac{c^{2} k_{z}^{2}}{4 \pi \sigma} / \int_{z_{t p}}^{z_{b}} \frac{d z}{v_{g, z}}
\end{aligned}
$$

where $v_{g, z}$ is the vertical group velocity, and in the second expression we have used constant wave energy flux in the propagation cavity, and the integral extends between the upper turning point $z_{t p}$ and the base of the crust $z_{b}$. This expression is simply interpreted as the local damping rate weighted by the time spent at that depth. For $k_{z} \gg k_{\perp}, v_{g, z} \propto k_{z}^{-1}$ so the ratio $z k_{z}^{3} / \rho^{1 / 3}$ determines the depth which dominates Ohmic decay (for impurity scattering). If $k_{z} \propto n_{e}^{1 / 2}$, the factor $n_{e}^{3 / 2} / \rho^{1 / 3}$ is dominated by the largest densities. We expect that up to an order unity prefactor, the Ohmic decay time for the mode is set by $\Omega \tau$ at the base of the crust, $\gamma^{-1} \approx P_{\text {Hall }}(\Omega \tau)_{b}$, where $(\Omega \tau)_{b} \approx B_{12} / Q$ when impurity scattering dominates (eq. [15).

\section{The elastic response of the crust to the wave}

The strain in the crust may be calculated by balancing the elastic and magnetic stresses, since ion inertia plays no role in the force balance for the low frequency Hall waves. This gives

$$
\theta=\frac{d \xi}{d z}=-\frac{B_{z}}{4 \pi \mu} \delta B
$$

We show the strain as a function of depth in the lower panels of Figures 11. 13. We write the shear modulus (Strohmayer et al. 1991) as $\mu \propto n_{e}^{4 / 3} \approx 10^{30} \mathrm{erg} \mathrm{cm}^{-3}\left(z / z_{b}\right)^{16 / 3}$, giving $\theta \propto$ $z^{-13 / 3}$ below the turning point, and $\theta \propto z^{-1 / 3}$ above the turning point. The maximum strain occurs at or above the turning point. Using the WKB scaling, we find that at the turning point,

$$
\theta_{\text {turn }}=3 \times 10^{-7} B_{12}^{2} n^{13 / 9} \frac{\delta B_{b}}{B},
$$

where $\delta B_{b}$ is the amplitude of the mode at the base.

\section{More general cases of Hall waves}

For a horizontal background field, or a non-vertically propagating wave on a vertical background field, the calculation of the elastic response of the crust is more complicated, and we leave this for future work. Here, we give the WKB scalings for waves on a uniform horizontal field $B=B \hat{e}_{x}$, and set $k_{y}=0$ for simplicity, $\delta B=\delta B(z) \exp \left(i k_{x} x-i \omega t\right)$. The perturbation equation is

$$
\frac{d^{2} \delta B_{z}}{d z^{2}}+k_{x}^{2} \delta B_{z}\left[\frac{\omega^{2}}{\nu^{2} k_{x}^{4}}-1\right]=0
$$

giving the WKB dispersion relation $\omega= \pm \nu k_{x}\left(k_{x}^{2}+k_{z}^{2}\right)^{1 / 2}$. For $k_{z} \gg k_{x}$, this gives $P_{\text {Hall }} \approx t_{\text {Hall }} /\left(n k_{x} H_{b}\right)$. The location of the turning point is $z_{\text {turn }} \approx 3 H_{b} / n^{1 / 5}$, or $\rho_{\text {turn }} Y_{e} \approx 2 \times$ $10^{12} \mathrm{~g} \mathrm{~cm}^{-3} / n^{4 / 5}$. The turning point for the modes is deeper in than the vertically-propagating case; we require $n \approx 10^{4}$ for $\rho_{\text {turn }} \approx 10^{9} \mathrm{~g} \mathrm{~cm}^{-3}$.

\section{Non-zero background current: waves and unstable modes}

Before ending this section, we discuss the case of a horizontal field in more detail. In several recent papers, Rheinhardt, Geppert, and collaborators have discussed a "Hall drift instability" (HDI) driven by curvature in the background field (Rheinhardt \& Geppert 2002, hereafter RG02; Geppert \& Rheinhardt 2002; Rheinhardt, Konenkov, \& Geppert 2003; Geppert, Rheinhardt, \& Gil 2003). In this section, we first discuss a simple example of the instability to elucidate the basic physics. We then discuss the mode spectrum, and the relevance of the instability for small scale Hall waves.

Following RG02, we study the stability of a plane-parallel horizontal field that depends only on height $z, B=B(z) \hat{e}_{x}$, with corresponding current density $J=(c / 4 \pi)(d B / d z) \hat{e}_{y}$. However, for simplicity and to highlight the essential physics, we consider the Hall terms only. The perturbations are of the form $\delta B(z) \exp \left(i k_{x} x+\sigma t\right)$ (we set $k_{y}=0$ since RG02 find that these are the fastest growing modes). Since the background field does not evolve due to the Hall term, the perturbed induction equation is $\sigma \delta B=\nabla \times\left(\delta v_{e} \times B\right)+\nabla \times\left(v_{e} \times \delta B\right)$, or

$$
\left(\sigma+v_{e} \cdot \boldsymbol{\nabla}\right) \delta B=(B \cdot \boldsymbol{\nabla}) \delta v_{e}+(\delta B \cdot \boldsymbol{\nabla}) v_{e}-\left(\delta v_{e} \cdot \boldsymbol{\nabla}\right) B
$$

where $v_{e}=-J / n_{e} e$ is the electron velocity, and $\delta J=$ $(c / 4 \pi)(\nabla \times \delta B)$.

Writing an equation for $\delta B_{z}$, we find the generalization of equation (55) for this case is,

$$
\frac{\partial^{2} \delta B_{z}}{\partial z^{2}}=k_{x}^{2} \delta B_{z}\left[1+\frac{d v_{e} / d z}{\nu k_{x}^{2}}+\frac{\sigma^{2}}{\left(\nu k_{x}^{2}\right)^{2}}\right] .
$$

The eigenvalue $\sigma^{2}$ is purely real ${ }^{5}$ : the solution is either a pure growing/decaying mode, or oscillatory. Making a WKB approximation, $\partial / \partial z \rightarrow i k_{z}$, we find (writing $k^{2}=k_{x}^{2}+k_{z}^{2}$ ),

$$
\sigma^{2}=-\left(\nu k k_{x}\right)^{2}-\nu k_{x}^{2} \frac{d v_{e}}{d z}
$$

\footnotetext{
${ }^{5}$ To show this, multiply equation 57 by the complex conjugate $\delta B_{z}^{\star}$, and subtract the complex conjugate of equation 57 multiplied by $\delta B_{z}$. Integrating over volume, and assuming the boundary conditions are such that the boundary term vanishes, gives $\left(\sigma^{2}-\sigma^{2 \star}\right) \int d V\left|\delta B_{z}\right|^{2} / \nu^{2} k_{x}^{2}=0$.
} 
The first term is the usual Hall waves, $\sigma= \pm i \nu k k_{x}$; the second term depends on the electron velocity gradient, and leads to instability $\left(\sigma^{2}>0\right)$ if the electron velocity gradient is sufficiently negative, and for sufficiently long wavelengths,

$$
k^{2}<-\frac{1}{\nu} \frac{d v_{e}}{d z} .
$$

A more general integral criterion can be derived by multiplying equation (57) by $\delta B_{z}$ and integrating over the volume,

$$
\begin{aligned}
\sigma^{2} \int d V \frac{\left|\delta B_{z}\right|^{2}}{\nu^{2} k_{x}^{2}}=-\int & d V\left(\left|\delta B_{z}^{\prime}\right|^{2}+k_{x}^{2}\left|\delta B_{z}\right|^{2}\right) \\
& -\int d V\left|\delta B_{z}\right|^{2} \frac{d v_{e} / d z}{\nu},
\end{aligned}
$$

showing that $d v_{e} / d z$ must be sufficiently negative somewhere in the volume to lead to instability $\left(\sigma^{2}>0\right)$. We have numerically integrated equation (57) for the simple case of constant $n_{e}$ and a quadratic dependence of $B$ on depth, confirming the scalings of equations (58) and (59).

The basic physics of the instability can be understood by stepping through the evolution of an initial vertical perturbation $\delta B_{z}$, and recalling that the field is advected by the electron flow. First consider short wavelengths where the $d v_{e} / d z$ term is unimportant. The current perturbation $\delta J_{y}=-i k_{x} \delta B_{z} c / 4 \pi$ corresponds to a sheared electron flow, which gives rise to a perpendicular field component $\sigma \delta B_{y}=-i k_{x} B \delta v_{e, y}$ through the first term on the right hand side of equation (56). Associated with $\delta B_{y}$ is a current $\delta J_{z}=i k_{x} \delta B_{y} c / 4 \pi$, which in turn acts back on $\delta B_{z}, \sigma \delta B_{z}=-i k_{x} B \delta v_{e, z}$, thereby driving the Hall wave. When the $d v_{e} / d z$ term dominates, the first stage of this evolution is different. Shearing of the initial perturbation by the background electron flow generates $\delta B_{y}$ in the first step, $\sigma \delta B_{y}=-\delta B_{z}\left(d v_{e} / d z\right)$, transferring energy from the background field into the perturbation. Equation (58) shows the competition between the internal and external shearing, measured by the ratio of Hall wave frequency to the shear frequency $d v_{e} / d z$.

The criterion for instability we derive above generalizes that of RG02 to include the density variation with depth (see also Rheinhardt et al. 2003), and clarifies the nature of the instability: a background shear in the electron velocity drives growth of long wavelength perturbations whose characteristic Hall frequencies are less than $d v_{e} / d z$. Short wavelength Hall waves, with frequencies greater than $d v_{e} / d z$, are unaffected. This differs from the conclusions of RG02, who argue that HDI may be responsible for the growth of small-scale perturbations reported in some numerical simulations (e.g. Shalybkov \& Urpin 1997; Hollerbach \& Rüdiger 2002). Perhaps numerical instabilities are rather responsible for the excess small scale power 6 .

Is the HDI relevant for the evolution of field in the crust? If the picture of a turbulent Hall cascade is correct, the instability probably does not change the long-term evolution of the

\footnotetext{
${ }^{6}$ We found such an instability in trying to develop a 3D code to simulate the Hall effect. It is straightforward to see this in 1D in Cartesian coordinates. Using second-order centered differences in space and explicit time-stepping (first order, or, using Runga-Kutta, second order accurate), one can analytically show that all wavelengths are unstable! The growth rate of the instability for wavenumber $k$ on a grid of spacing $\Delta x$ scales as $\propto(k \Delta x)^{8}$, implying large lengthscale waves grow only after many box crossings, but grid-scale waves have a growth rate of order the time step. Including Ohmic diffusion only cures this instability if a prohibitively short time step is used. In 1D, the instability is easily fixed by using implicit time-stepping, but this cannot be simply extended to 3D using operator splitting.
}

field, since intermediate scales will "fill in" as the cascade develops. If the background field varies on a lengthscale $H$, the most unstable modes are those with the largest $k_{x}$ compatible with equation (59), implying $k_{x} \sim 1 / H$, or corresponding to spherical harmonic $l$ of tens. Rheinhardt et al. (2003) then argue that an initially dipolar field will develop small latitudinal scale structure; however, this conclusion is sensitive to the initial distribution of currents in the crust. An additional point is that changes in the crustal field may not propagate to the surface because of the very efficient reflection at the solid/fluid interface (see discussion in the Appendix). Therefore predictions of complex surface fields produced by HDI (Geppert et al. 2003) may be premature.

\subsection{Nonlinear Coupling of Oscillation Modes}

We now discuss the implications of our results for the turbulent Hall cascade suggested by GR. They estimated the nonlinear transfer time between different modes to be longer than the mode period, and proposed that the cascade would be a weak cascade. In this case, the expected scaling of magnetic energy is $B^{2}(k) \propto k^{-2}$, with a cutoff due to Ohmic dissipation at a scale $\lambda \sim L / \Omega \tau$, where $L$ is the scale on which the turbulence is being driven. However, the simulations of Biskamp et al. (1999) show a different scaling, $B^{2}(k) \propto k^{-7 / 3}$ appropriate for a strong cascade (to derive this scaling, follow the argument of GR $\$ 4$, but choosing their eq. [47] rather than eq. [48]). In this case, the Ohmic cutoff is for $\lambda \sim L /(\Omega \tau)^{3 / 2}$. The difference arises most likely because of GR's assumption of isotropy: a strong cascade implies mode periods longer than the non-linear transfer time, which suggests a cascade perpendicular to the magnetic field direction, for which the Hall wave periods are long. Unfortunately, Biskamp and coauthors did not present an analysis of anisotropy in their numerical results. Such a study would be extremely interesting.

How many modes are involved in the cascade? If the energy transfer is limited by Ohmic dissipation, then for a strong cascade we find

$$
n_{\max } \approx(\Omega \tau)^{3 / 2} \approx 3 \times 10^{4}\left(\frac{B_{12}}{Q_{-3}}\right)^{3 / 2}
$$

where we use the result for impurity scattering for $\Omega \tau$. The turning point for the mode with $n=n_{\max }$ (assuming vertical propagation and a vertical field) is at $\rho_{\max } \approx$ $10^{6} \mathrm{~g} \mathrm{~cm}^{-3}\left(Q_{-3} / B_{12}\right)^{2}$.

In $\$ 4.3$ we calculated the maximum strain $\theta_{\max }$ for a Hall wave with given $n$ and amplitude at the base $\delta B_{b}$. As $n$ increases, $\theta_{\max }$ increases, implying that the crust will yield for large enough $n$. However, this increase with $n$ is offset by the decreasing amplitude $\delta B_{b}$ with $n$ if there is a turbulent cascade: for a strong (weak) cascade, $\delta B \propto n^{-7 / 6}\left(\propto n^{-1}\right)$. The value of strain at which the crust will yield $\theta_{\text {yield }}$ is uncertain (e.g. Ruderman 1976), but probably lies in the range $\theta_{\text {yield }} \sim 10^{-5}-10^{-2}$. For the weak cascade, $\theta_{\max }$ decreases more slowly with $n$. Using equation (54) for vertically-propagating waves, we find for this case that the crust will yield for modes with

$$
n \gtrsim 6 \times 10^{5}\left(\frac{\theta_{\text {yield }}}{10^{-4}}\right)^{\beta} B_{12}^{-2 \beta}\left(\frac{\delta B_{b, 0}}{B}\right)^{-\beta},
$$

where $\delta B_{b, 0}$ is the amplitude of the mode at the largest lengthscale, and $\beta=9 / 4$. For a strong cascade, the prefactor is $2 \times 10^{9}$ (corresponding to $\Omega \tau \approx 10^{6}$ ) and $\beta=3.2$. Given our 
estimate for $n_{\max }$ (eq. [61]), we therefore do not expect the cascade to be limited by crust yielding, but rather Ohmic dissipation. However, we stress again that we have not yet calculated the crust response for arbitrary wave propagation and background field directions. Therefore, equation (62) should be taken as an initial estimate only.

\section{SUMMARY AND CONCLUSIONS}

In this paper, we have addressed various aspects of magnetic field evolution in neutron star crusts. Our aim has been to place previous studies of the physics of the Hall effect in context: first, by addressing when and for which neutron stars the Hall effect is important relative to Ohmic decay; second, by calculating the allowed "initial conditions" by following the Ohmic decay prior to dominance of the Hall effect; third, by discussing for the first time the properties of Hall waves in a realistic crust model, and the implications for the Hall cascade.

We now summarise our main results and conclusions:

(i) The relative importance of Ohmic decay and the Hall effect is summarized in Figure 4 which shows the $\Omega \tau=1$ contour in the $B-T$ plane. In isolated neutron stars with $B \lesssim 10^{13} \mathrm{G}$, the dominance of the Hall effect depends sensitively on the impurity parameter $Q$ : recent calculations by Jones (2001) indicate $Q \gtrsim 1$ for densities greater than neutron drip (see also de Blasio 2000 for densities lower than neutron drip), in which case the Hall effect never dominates the evolution of crustal currents, considerably simplifying the evolution. If $Q \sim 10^{-3}$ however, as in the original Flowers \& Ruderman (1977) estimate, Ohmic decay dominates the Hall effect only for a time $t_{\text {switch }} \approx 2 \times 10^{4}$ years $/ B_{12}^{3}$, after which the conductivity is set by impurity scattering, giving $\Omega \tau \approx B_{12} / Q$. In accreting neutron stars with $B \lesssim 10^{13} \mathrm{G}$, Ohmic decay always dominates, since the crust is heated $\left(T \gtrsim 10^{8} \mathrm{~K}\right.$, e.g. Brown 2000) and may also be impure $Q \gtrsim 1$ (Schatz et al. 1999).

(ii) Figure 9 summarizes the evolution of currents in the crust while Ohmic decay dominates $\left(t<t_{\text {switch }}\right)$. The electrical conductivity at these early times is set by phonon scattering. This figure relates previous work on either Ohmic decay eigenmodes, or on self-similar solutions, in a simple way. For a particular time $t$, Figure 9 gives the density $\rho_{c}$ down to which the currents have significantly decayed. For $\rho>\rho_{c}$, no significant evolution of the currents has occurred. For $\rho<\rho_{c}$, the current distribution is self-similar, being dominated by the Ohmic decay mode with turning point at $\rho=\rho_{c}$. Because $t_{\text {switch }} \propto 1 / B^{3}$, the allowed distribution of currents when the Hall effect begins to dominate is very sensitive to $B$. For $B \approx 10^{13} \mathrm{G}, \rho_{c}$ lies at or above the top of the crust; for $B \approx 10^{11} \mathrm{G}, \rho_{c}$ lies at the base of the crust. At late times, when $\rho_{c}$ is greater than the initial location of the currents, the current distribution evolves in a self-similar manner and the surface field decays as a power law (eq. 31]).

(iii) The basic physics of the Hall effect is that the field in the crust is advected by the electron flow. This is summarized in Figure 1 which considers a variant of the usual laboratory demonstration of the Hall effect. Figure 10 shows the evolution of an initial poloidal dipole field, in which the toroidal currents initially "twist" the field; the resulting poloidal currents then generate a quadrupole poloidal field via a "pinch". These considerations led us to a simple time-independent solution: a poloidal dipole field, in which the electrons rotate rigidly, does not evolve due to the Hall effect. (iv) We discussed the properties of Hall waves in the crust. The simplest example is a background magnetic field which is uniform and vertical (for example, emerging from the core). The vertical wavelength of the wave grows towards the top of the crust (as pointed out by GR), and we showed that the turning point location is $\rho_{\text {turn }} \approx 10^{12} \mathrm{~g} \mathrm{~cm}^{-3} / n^{4 / 3}$. Below the turning point, the wave is able to propagate and its properties are well-described by WKB scalings. Above the turning point, the wave evanesces towards the top of the crust.

(v) A horizontal field (e.g. as may be produced if the currents supporting the field are predominantly in the crust) has similar wave-like solutions at short wavelengths. However, if there is a background current (the background $B$ varies with height), long wavelength perturbations become unstable. The physics of this instability is that the shear in the background electron velocity drives unstable growth, overcoming the internal shear of the wave which would otherwise drive an oscillation. Therefore we agree with the recent work of Geppert and collaborators that there is an instability, and we have clarified its basic physics. However, we argued that the relevance of this instability for the field evolution in the crust, and particularly for the observable surface field, is not clear.

(vi) We have made a first attempt to address the question of whether Hall waves strain the crust beyond its yield point. We find that the maximum strain is at or above the turning point for the mode (eq. [54). However, our calculation is limited to the special case of vertically-propagating waves on a vertical background field; we leave further calculations of the response of the crust to non-vertical background fields and wavevectors for future work. In particular, the force balance is important to consider for magnetar strength fields, for which $B^{2}>4 \pi \mu$, directly coupling the induction and momentum equations in the crust (see the recent discussion in Arras et al. 2004).

(vii) Finally, we briefly discussed the non-linear evolution. Recent numerical work on Whistler turbulence (Biskamp et al. 1999) has demonstrated GR's conjecture that the Hall effect would create a "cascade" of energy to small scales. However, the scalings of the cascade imply strong, anisotropic turbulence rather than weak, isotropic turbulence as assumed by GR. The Hall wave eigenfunctions calculated in $\$ 4$ (again, for vertically propagating waves and a vertical field) show that crust breaking is probably not important for standard radio pulsar strength fields.

We thank Omer Blaes, Phil Chang, Lars Bildsten, Roger Romani, Chris Thompson, Steve Thorsett, and Dmitry Uzdensky for useful discussions, and Peter Goldreich for stressing the simple physics that the magnetic field moves with the electrons. AC acknowledges support from NASA through Hubble Fellowship grant HF-01138 awarded by the Space Telescope Science Institute, which is operated by the Association of Universities for Research in Astronomy, Inc., for NASA, under contract NAS 5-26555. PA acknowledges support from the Canadian Institute for Theoretical Astrophysics. PA is an NSF Astronomy and Astrophysics Postdoctoral Fellow. This material is based upon work supported by the National Science Foundation under Grant No. 0201636 to the University of California at Santa Barbara and Grants AST-0328821 and PHY-0215581 to the University of Wisconsin at Madison. 


\section{APPENDIX}

\section{BOUNDARY CONDITIONS FOR HALL WAVES}

In this Appendix we discuss the boundary conditions for Hall waves in the crust. First we argue that the disparity in propagation speeds between solid and liquid implies near total reflection of wave energy at the boundaries. We present the results of simple toy models to quantify the transmission coefficient. We then derive the boundary conditions. Lastly we specialize the boundary conditions for vacuum solutions in the liquid.

For elastic response of the crust, i.e. neglecting irreversible processes such as plastic flow, we naively expect Hall wave energy to be almost perfectly trapped in the crust. For a wavelength of order the size of the crust, the Hall wave propagation speed is vastly smaller than the Alfven speed, implying a large impedence mismatch between the solid crust, liquid core and liquid ocean.

To get an analytic handle on the size of the reflection and transmission coefficients, consider a region with uniform density, constant vertical background field, crust with constant shear modulus for $z<0$ and ocean with zero shear modulus for $z>0$ (see Blaes et al. 1989 for the Alfven wave case). This toy problem is of limited validity, since the density is not constant, and also since in reality the WKB assumption is violated (vertical wavelength in the ocean $\gg$ scale height), but we can gain useful intuition without much effort. We consider a vertically propagating Hall wave incident from below, and then include all possible reflected and transmitted waves. The two circular polarization states are decoupled in the boundary conditions, hence we need only include reflected Hall and elastic-Alfven waves and a transmitted Alfven wave. We use the boundary conditions derived below to determine the amplitudes of reflected and transmitted waves. We leave this straightforward calculation as an exercise for the reader, and present the results. In the limit $B^{2} \ll 4 \pi \mu$ near the top of the crust, we find the amplitudes of the reflected Hall, reflected elastic-Alfven, and transmitted Alfven waves are

$$
\begin{aligned}
\delta B_{(\text {ref Hall })} & \simeq-\left(1+\frac{v_{\text {Hall }}}{v_{\text {Alfven }}}\right) \delta B_{\text {(inc Hall })} \\
\delta B_{(\text {ref Alfven })} & \simeq-\frac{v_{\text {Hall }} v_{\text {Alfven }}}{v_{\text {shear }}^{2}} \delta B_{\text {(inc Hall })} \\
\delta B_{\text {(tran Alfven })} & \simeq-\frac{v_{\text {Hall }}}{v_{\text {Alfven }}} \delta B_{(\text {inc Hall })},
\end{aligned}
$$

yielding an energy transmission coefficient

$$
T=2 v_{\text {Hall }} / v_{\text {Alfven }} \simeq 2.4 \times 10^{-12}\left[B_{z, 12} P(\mathrm{yr})\right]^{-1 / 2} .
$$

Hence Hall waves experience negligible damping due to wave leakage in this simple case. Interestingly, we find that both the reflected and transmitted Alfven waves have a very small amplitude compared to the Hall wave. In this simple toy problem, the (observable) field in the liquid is composed of the constant background field (generated in the core) as well as the perturbation. Since the amplitude of the perturbation is infinitesimally small in the liquid as compared to the solid, the observable external field may be somewhat different than the field in the crust. If this behavior persists for more realistic field distributions, the observable external field may not be the same as the field in the crust; the neutron star is in effect "hiding" its field. This effect is of course larger when the currents are mainly in the crust rather than the core.

If we relax the restriction of vertical propagation (i.e. allow finite $k_{\perp}$ ), there is a qualitative change in the nature of the waves. The low Hall wave frequency implies the reflected elastic-Alfven wave becomes evanescent with e-folding distance $\sim k_{\perp}^{-1}$, and the transmitted Alfven wave has wavevector nearly perpendicular to the background magnetic field. Both polarization states must now be included for the reflected elastic-Alfven wave and transmitted Alfven wave. In addition, the two polarizations of reflected Hall wave correspond to propagating and evanescent waves. With so many waves included, the analysis is complicated, and a nontrivial check of both our numerical results and the boundary conditions derived below was that energy was conserved over a large range in frequency and $k_{\perp}$. For incident Hall wave of amplitude unity, the numerical results can be summarized as : (i) the amplitude of the reflected propagating Hall wave is $1+\mathcal{O}\left(k_{\perp} / k_{\mathrm{z}, \mathrm{Hall}}\right)$, (ii) the amplitude of all other reflected waves scales as $\mathcal{O}\left(k_{\perp} / k_{\mathrm{z}, \text { Hall }}\right)$, (iii) the transmission coeffient is independent of $k_{\perp}$, and agrees in order of magnitude with eq A2 Hence even in this more general case the crust still acts as a nearly perfect resonant cavity, and the wave is essentially unobservable since it's amplitude in the liquid is so small.

We now discuss the boundary conditions. We do not linearize the magnetic field, but for simplicity we use the linearized shear stress. We use the notation $[f]=(f)_{\text {solid }}-(f)_{\text {liquid }}$, and the normal to the boundary is in the $z$ direction. By integrating over a small region near the solid-liquid boundary we find

$$
\begin{array}{ll}
{\left[B_{z}\right]=0} & \text { from } \boldsymbol{\nabla} \cdot B=0 \\
{\left[J_{z}\right]=0} & \text { from } \boldsymbol{\nabla} \cdot J=0 \\
{\left[\xi_{z}\right]=0} & \text { from finiteness of } \boldsymbol{\nabla} \cdot \boldsymbol{\xi} \\
{\left[(K-2 \mu / 3) \nabla \cdot \boldsymbol{\xi} \delta_{i z}+\mu\left(\xi_{i, z}+\xi_{z, i}\right)+(4 \pi)^{-1}\left(B_{i} B_{z}-\frac{1}{2} B^{2} \delta_{i z}\right)\right]+g \xi_{z}[\rho] \delta_{i z}=0} & \text { from momentum equation } \\
{\left[-\frac{1}{c}\left\{\left(\dot{\xi}-\frac{J}{n_{e} e}\right) \times B\right\}_{\perp}+\frac{J_{\perp}}{\sigma}\right]=0} & \text { from induction equation. }
\end{array}
$$

The last term in equation (A6) arises from the buoyancy force $-e_{r} g \delta \rho=e_{r} g \nabla \cdot\left(\rho \xi_{z}\right)$, and we have ignored the perturbed gravitational potential. Equation A5 is required since $\nabla \cdot \xi$ would be infinite if $\xi_{z}$ was discontinuous across an infinitesimally thin layer. 
The Hall effect, like Ohmic diffusion, involves a higher spatial derivative than the ion advection term in the induction equation. Hence an additional boundary condition comes from the requirement that these terms remain finite in equation (A7). If the magnetic field was discontinuous across the effectively infinitely thin boundary, the Hall and Ohmic electric fields would diverge. Hence we must add the boundary condition

$$
\left[B_{\perp}\right]=0 \quad \text { from finite Hall voltage. }
$$

This boundary condition is similar to the no-slip condition from viscous hydrodynamics ${ }^{7}$.

The horizontal stress boundary conditions can be greatly simplified to give a useful result for the strain. Taken together, equations $\mathrm{A} 3$ and $\mathrm{A} 8$ imply $[B]=0$, which has the interesting consequence that the magnetic stress drops out of the boundary condition in eq Since $\mu=0$ in the liquid, the horizontal components of the (symmetrized) strain must vanish on the solid side of the boundary,

$$
\left(\xi_{i, z}+\xi_{z, i}\right)_{\text {solid }}=0 \quad \text { for } i=x, y \text {. }
$$

As the magnetic field in the crust evolves by the Hall effect, equation (A9) is not satisfied in general over the entire crust. As the wave approaches the boundary, it must adjust in order to satisfy this condition. On qualitative grounds, we expect this would reflect the magnetic energy even if the wave is not short lengthscale. This result suggests that even for the fully nonlinear case, if the currents are generated in the crust there may be a significant difference between crustal field and observable external field.

As discussed by Kingsep et al. (1990), the higher spatial derivatives give rise to a boundary layer effect in Hall-MHD (without the elastic shear stress), with ion advection dominating on large scales and the Hall effect on small scales (shorter than the ion penetration depth). As a test of the boundary conditions, we have redone the transmission-reflection problem of Blaes et al. (1989) for Alfen waves to escape from the crust, now including the Hall term in the induction equation. We find that for typical Alfven wave frequencies we recover the Blaes et al. (1989) Alfven-wave transmission coefficient, consistent with the idea that the Hall term only affects Alfven waves quite close to the boundary, but cannot alter a global property such as transmission out of the crust.

Lastly, we note that if the currents are small in the liquid (say due to low conductivity), or equivalently if the field is near the vacuum solution, one finds an interesting boundary condition on the field in the solid. Since $J_{z}=0$ in the liquid, and $\left[J_{z}\right]=0$, $J_{z} \propto k_{x} B_{y}-k_{y} B_{x}=0$ in the solid also. We can always choose $k_{y}=0$ without loss of generality, again implying $B_{y}=0$ in the solid at the boundary.

\section{REFERENCES}

Arras, P., Cumming, A., \& Thompson, C. 2004, ApJL, submitted

Baiko, D. A., \& Yakovlev, D. G. 1995, Ast. Lett., 21, 702

Baiko, D. A., \& Yakovlev, D. G. 1996, Ast. Lett., 22, 708

Bhattacharya D., 1995, in X-Ray Binaries, ed. W. H. G. Lewin, J. van

Paradijs, \& E. P. J. van den Heuvel (Cambridge: Cambridge University Press), 233

Bhattacharya D., \& Srinivasan, G. 1995, in X-Ray Binaries, ed. W. H. G. Lewin, J. van Paradijs, \& E. P. J. van den Heuvel (Cambridge: Cambridge University Press), 495

Bhattacharya, D., Wijers, R. A. M. J., Hartman, J. W., \& Verbunt, F. 1992, ApJ, 254, 198

Biskamp, D., Schwarz, E., Zeiler, A., Celani, A., \& Drake, J. F. 1999, Phys. Plasmas, 6, 751

Blaes, O., Blandford, R., Goldreich, P. \& Madau, P. 1989, ApJ, 343, 839.

Blandford, R. D., Applegate, J. H. \& Hernquist, L. 1983, MNRAS, 204, 1025.

Brown, E. F. 2000, ApJ, 531, 988

Chakrabarty, D., Morgan, E. H., Muno, M. P., Galloway, D. K., Wijnands, R., van der Klis, M., \& Markwardt, C. B. 2003, Nature, 424, 42

Chandrasekhar, S., 1961, Hydrodynamic and Hydromagnetic Stability,

(International Series of Monographs on Physics, Oxford: Clarendon)

Colpi, M., Geppert, U., \& Page, D. 2000, ApJ, 529, L29

Cordes, J. M., \& Chernoff, D. F. 1998, ApJ, 505, 315

Cumming, A., Zweibel, E. G., \& Bildsten, L. 2001, ApJ, 557, 958

De Blasio, F. V. 2000, A\&A, 353, 1129

Douchin, F., \& Haensel, P. 2001, A\&A, 380, 151

Duncan, R. C. \& Thompson, C. 1992, ApJ, 392, L9

Eichler, D. \& Cheng, A.F. 1989, ApJ, 336, 360.

Ferraro, V. C. A. 1937, MNRAS, 97, 458

Flowers, E., \& Ruderman, M. A. 1977, ApJ, 215, 302

Franco, L.M., Link, B., \& Epstein, R.I. 2000, ApJ, 543, 987

Gavriil, F. P., Kaspi, V. M., \& Woods, P. M. 2002, Nature, 419, 142

Geppert, U. \& Rheinhardt, M. 2002, A\&A, 392, 1015

Geppert, U., Rheinhardt, M., \& Gil, J. 2003, A\&A, submitted astro-ph/0311121,

Geppert, U. \& Urpin, V. 1994, MNRAS, 271, 490

Gnedin, O. Y., Yakovlev, D. G., \& Potekin, A. Y. 2001, MNRAS, 324, 725

Goldreich, P., \& Reisenegger, A. 1992, ApJ, 395, 250 (GR)

Gonthier, P. L., Ouellette, M. S., Berrier, J., O'Brien, S., \& Harding, A. K. 2002, ApJ, 565, 482

Gunn, J. E., \& Ostriker, J. P. 1970, ApJ, 160, 979

7 At present, we have no boundary condition on $\boldsymbol{\xi}_{\perp}$. Including a viscous shear stress would require $\left[\boldsymbol{\xi}_{\perp}\right]=0$.
Haensel, P., \& Pichon, B. 1994, A\&A, 283, 313

Haensel, P., \& Zdunik, J. L. 1990, A\&A, 229, 117

Heyl, J. S., \& Kulkarni, S. R. 1998, ApJ, 506, L61

Hollerbach, R. \& Rüdiger, G. 2002, MNRAS, 337, 216

Itoh, N., \& Kohyama, Y. 1993, ApJ, 404, 268

Jackson, J.D. 1975, "Classical Electrodynamics", New York: Wiley, 1975, 2nd ed.

Jones, P. B. 1988, MNRAS, 233, 875

Jones, P. B. 2001, MNRAS, 321, 167

Konar, S. \& Bhattacharya, D. 1997, MNRAS, 284, 311

Kingsep, A. S., Chukbar, K. V., \& Yan'kov, V. V. 1990, Rev. Plasma Phys., 16,243

Mackie, F. D., \& Baym, G. 1977, Nucl. Phys. A, 285, 332

Mestel, L. 1999, "Stellar Magnetism" (Oxford University Press)

Muslimov, A. G. 1994, MNRAS, 267, 523

Naito, T., \& Kojima, Y. 1994, MNRAS, 266, 597

Narayan, R., \& Ostriker, J. P. 1990, ApJ, 352, 222

Negele, J. W., \& Vautherin, D. 1973, Nucl. Phys. A, 207, 298

Ostriker, J. P., \& Gunn, J. E. 1969, ApJ, 157, 1395

Page, D., Geppert, U., \& Zannias, T. 2000, A\&A, 360, 1052

Pethick, C. J., \& Sahrling, M. 1995, ApJ, 453, L29

Potekhin, A. Y., Baiko, D. A., Haensel, P., \& Yakovlev, D. G. 1999, A\&A, 346, 345

Potekhin, A. Y. \& Chabrier, G. 2000, Phys. Rev. E, 62, 8554

Rheinhardt, M., \& Geppert, U. 2000, Phys. Rev. Lett., 88, 101103

Rheinhardt, M., Konenkov, D., \& Geppert, U. 2003, A\&A, submitted (astro-ph/0310917,

Ruderman, M. 1976, ApJ, 203, 213

Ruderman, M., Zhu, T., \& Chen, K. 1998, ApJ, 492, 267

Sang, Y., \& Chanmugam, G. 1987, ApJ, 323, L61

Schatz, H., Bildsten, L., Cumming, A., \& Wiescher, M. 1999, ApJ, 524, 1014 Shalybkov, D. A., \& Urpin, V. A. 1997, A\&A, 321, 685

Shapiro, S. L., \& Teukolsky, S. A. 1983, "Black Holes, White Dwarfs, and Neutron Stars: The Physics of Compact Objects" (Wiley)

Strohmayer, T., Van Horn, H.M., Ogata, S., Iyetomi, H. \& Ichimaru, S. 1991, ApJ, 375, 679 .

Tauris, T. M., \& Konar, S. 2001, A\&A, 376, 543

Tauris, T. M., \& Manchester, R. N. 1998, MNRAS, 298, 625

Thompson, C. \& Duncan, R. C. 1995, MNRAS, 275, 255

Thompson, C. \& Duncan, R. C. 1996, ApJ, 473, 322 
Trümper, J., Pietsch, W., Reppin, C., Voges, W., Staubert, R., \& Kendziorra, E. 1978, ApJ, 219, L105

Urpin, V. A., Chanmugam, G., \& Sang, Y. 1994, ApJ, 433, 780

Urpin, V., Geppert, U., \& Konenkov, D. 1998, MNRAS, 295, 907

Urpin, V. \& Konenkov, D. 1997, MNRAS, 284, 741
Urpin, V. A. \& Muslimov, A. G. 1992, MNRAS, 256, 261

Urpin, V. A., \& Shalybkov, D. 1999, MNRAS, 304, 451

Vainshtein, S. I., Chitre, S. M., \& Olinto, A. V. 2000, Phys. Rev. E, 61, 4422

Watson, G.N. 1966, "Theory of Bessel Functions", Cambridge University

Press 